\title{
Invariance Principle for the Random Conductance Model with dynamic bounded Conductances
}

\author{
Sebastian Andres *
}

\begin{abstract}
We study a continuous time random walk $X$ in an environment of dynamic random conductances in $\mathbb{Z}^{d}$. We assume that the conductances are stationary ergodic, uniformly bounded and bounded away from zero and polynomially mixing in space and time. We prove a quenched invariance principle for $X$, and obtain Green's functions bounds and a local limit theorem. We also discuss a connection to stochastic interface models.
\end{abstract}

Keywords: Random conductance model, dynamic environment, invariance principle, ergodic, corrector, point of view of the particle, stochastic interface model.

Subject Classification: 60K37, 60F17, 82C41

\section{Introduction}

We consider the Euclidean lattice $\mathbb{Z}^{d}$ equipped with the set $E_{d}$ of non oriented nearest neighbour bonds: $E_{d}=\left\{e=\{x, y\}: x, y \in \mathbb{Z}^{d},|x-y|=1\right\}$. We will also write $x \sim y$ when $\{x, y\} \in E_{d}$. Denote by $\hat{\Omega}=[0, \infty)^{E_{d}}$ and by $\Omega$ the set of all measurable functions from $\mathbb{R}$ to $\hat{\Omega}$. We equip $\Omega$ with a $\sigma$-algebra $\mathcal{F}$ and a probability measure $\mathbb{P}$ so that $(\Omega, \mathcal{F}, \mathbb{P})$ becomes a probability space. The random environment is given by the coordinate maps $\mu_{e}^{\omega}(t)=\omega_{e}(t), t \in \mathbb{R}, e \in E_{d}$. We will refer to $\mu_{e}(t)$ as the conductance of the edge $e$ at time $t$. Further, write $\mu_{x y}^{\omega}(t)=\mu_{\{x, y\}}(t)=\mu_{y x}(t)$, and $\mu_{x y}(t)=0$ if $\{x, y\} \notin E_{d}$, and set

$$
\mu_{x}(t)=\sum_{y \in \mathbb{Z}^{d}} \mu_{x y}(t)=\sum_{y \sim x} \mu_{x y}(t) .
$$

We denote by $D\left(\mathbb{R}, \mathbb{Z}^{d}\right)$ the space of $\mathbb{Z}^{d}$-valued càdlàg functions on $\mathbb{R}$. For a given $\omega \in \Omega$ and for $s \in \mathbb{R}$ and $x \in \mathbb{Z}^{d}$, let $P_{s, x}^{\omega}$ be the probability measure on $D\left(\mathbb{R}, \mathbb{Z}^{d}\right)$, under which the coordinate process $\left(X_{t}\right)_{t \in \mathbb{R}}$ is the continuous-time Markov chain on $\mathbb{Z}^{d}$ starting in $x$ at time $t=s$ with time-dependent generator given by:

$$
\mathcal{L}_{t}^{\omega} f(x)=\sum_{y \sim x} \mu_{x y}^{\omega}(t)(f(y)-f(x))
$$

*Research partially supported by NSERC (Canada) 
That is, $X$ is the time-inhomogeneous random walk, whose time-dependent jump rates are given by the conductances. Note that the counting measure, independent of $t$, is an invariant measure for $X$. Further, we denote by $p^{\omega}(s, x ; t, y), x, y \in \mathbb{Z}^{d}, s \leq t$, the transition densities of the time-inhomogeneous random walk $X$. This model of a random walk in a random environment is known in the literature - at least in the case of timeindependent conductances - as the Random Conductance Model or RCM. Note that the total jump rate out of any site $x$ is not normalized, in particular the sojourn time at site $x$ depends on $x$. Therefore, the random walk $X$ is sometimes called the variable speed random walk (VSRW). However, for the purpose of this paper it would also be possible to consider the constant speed random walk (CSRW) with total jump rates normalized to one (cf. Remark 1.5 below).

On $(\Omega, \mathcal{F}, \mathbb{P})$ we define a $d+1$ parameter group of transformations $\left(\tau_{t, x}\right)_{(t, x) \in \mathbb{R} \times \mathbb{Z}^{d}}$ by

$$
\tau_{t, x}: \Omega \rightarrow \Omega \quad\left(\mu_{e}(s)\right)_{s \in \mathbb{R}, e \in E_{d}} \mapsto\left(\mu_{x+e}(t+s)\right)_{s \in \mathbb{R}, e \in E_{d}}
$$

so that obviously $\tau_{s+t, x+y}=\tau_{s, x} \circ \tau_{t, y}$. Notice that

$$
p^{\tau_{h, z} \omega}(s, x ; t, y)=p^{\omega}(s+h, x+z ; t+h, y+z), \quad \mu_{x y}^{\tau_{h, z} \omega}(t)=\mu_{x+z, y+z}^{\omega}(t+h) .
$$

We are interested in the $\mathbb{P}$ almost sure or quenched long range behavior, in particular in obtaining a quenched functional limit theorem (QFCLT) or invariance principle for the process $X$ starting in 0 at time 0 . To that aim we need to state some assumptions on the environment measure $\mathbb{P}$.

Assumption A1 (Ergodicity). $\tau_{t, x}(A) \in \mathcal{F}$ for all $A \in \mathcal{F}$, and the measure $\mathbb{P}$ is invariant and ergodic w.r.t. $\left(\tau_{t, x}\right)$, i.e. $\mathbb{P}[A] \in\{0,1\}$ for any event $A$ such that $\tau_{t, x}(A)=A$ for all $t \in \mathbb{R}$ and $x \in \mathbb{Z}^{d}$.

Assumption A2 (Stochastic Continuity). For any $\delta>0$ and $f \in L^{2}(\mathbb{P})$ we have

$$
\lim _{h \rightarrow 0} \mathbb{P}\left[\left|f\left(\tau_{h, 0} \omega\right)-f(\omega)\right| \geq \delta\right]=0 .
$$

Thanks to Assumption A1 and A2 the family of operators $\left(T_{t}\right)_{t \in \mathbb{R}}$ acting on $L^{2}(\mathbb{P})$, defined by $T_{t} f=f \circ \tau_{t, 0}$, forms a strongly continuous group of unitary operators. Its $L^{2}(\mathbb{P})$-generator will be denoted by $D_{t}: \mathcal{D}\left(D_{t}\right) \rightarrow L^{2}(\mathbb{P})$, defined by

$$
D_{t} f(\omega)=\frac{\partial}{\partial t} T_{t} f_{\mid t=0}(\omega)=\frac{\partial}{\partial t}{ }_{\mid t=0} f\left(\tau_{t, 0} \omega\right) .
$$

By Corollary 1.1.6 in [EK] the generator is closed and densely defined. Note that $D_{t}$ is an anti-selfadjoint operator in $L^{2}(\mathbb{P})$, i.e.

$$
\left\langle D_{t} f, g\right\rangle_{\mathbb{P}}=-\left\langle f, D_{t} g\right\rangle_{\mathbb{P}}, \quad f, g \in \mathcal{D}\left(D_{t}\right)
$$

in particular

$$
\left\langle D_{t} f, f\right\rangle_{\mathbb{P}}=0, \quad f \in \mathcal{D}\left(D_{t}\right)
$$


Assumption A3 (Ellipticity). There exist positive constants $C_{l}$ and $C_{u}$ such that

$$
\mathbb{P}\left[C_{l} \leq \mu_{e}(t) \leq C_{u}, \forall e \in E_{d}, t \in \mathbb{R}\right]=1 .
$$

We recall that under Assumption A3 the following heat kernel estimates have been proven in [DD] (see also [GOS, Appendix B] for similar bounds).

Proposition 1.1. There exist constants $c_{1}, \ldots, c_{5}$ such that for $\mathbb{P}$-a.e. $\omega$ and for every $t \geq s \geq 0$ the following holds

i) If $x, y \in \mathbb{Z}^{d}$ and $D:=|x-y| \leq c_{1}(t-s)$, then

$$
\left.p^{\omega}(s, x ; t, y) \leq \frac{c_{2}}{(t-s)^{d / 2}} \exp \left(-c_{3} D^{2} /(t-s)\right) \quad \text { (Gaussian regime }\right)
$$

ii) If $x, y \in \mathbb{Z}^{d}$ and $D:=|x-y| \geq c_{1}(t-s)$, then

$$
p^{\omega}(s, x ; t, y) \leq \frac{c_{4}}{1 \vee(t-s)^{d / 2}} \exp \left(-c_{5} D(1+\log (D /(t-s))) \quad\right. \text { (Poisson regime). }
$$

Our first result is the following averaged or annealed FCLT. Let $\mathbb{P} \otimes P_{s, x}^{\omega}$ be the joint law of the environment and the walk, and the annealed law is defined to be the marginal $\mathbb{P}_{s, x}^{*}=\int_{\Omega} P_{s, x}^{\omega} d \mathbb{P}(\omega)$. Further, let

$$
X_{t}^{(\varepsilon)}=\varepsilon X_{t / \varepsilon^{2}}, \quad t \geq 0
$$

Theorem 1.2. Let $d \geq 1$ and suppose that Assumptions A1-A3 hold. Then, the law of $X^{(\varepsilon)}$ converges under $\mathbb{P}_{0,0}^{*}$ to the law of a Brownian motion on $\mathbb{R}^{d}$ with a deterministic non-degenerate covariance matrix $\Sigma$.

To prove a QFCLT we will need some mixing assumptions on the environment. We denote by $B(\Omega)$ the set of bounded and measurable functions on $\Omega$ and $C_{b \text {,loc }}^{1}(\hat{\Omega})$ the set of differentiable functions on $\hat{\Omega}=[0, \infty)^{E_{d}}$ with bounded derivatives depending only on a finite number of variables.

Assumption A4 (Time-mixing of the environment). There exists $p_{1}>1$ such that for every $m \in \mathbb{N}$ the following holds: For each $\varphi, \psi \in B(\Omega)$ of the form $\varphi(\omega)=\tilde{\varphi}\left(\omega\left(t_{1}\right)\right)$ and $\psi(\omega)=\tilde{\psi}\left(\omega\left(t_{2}\right)\right)$, where $\left|t_{1}-t_{2}\right| \geq 1$ for some $\tilde{\varphi}, \tilde{\psi} \in C_{b, \text { loc }}^{1}(\hat{\Omega})$ depending on $m$ variables we have

$$
|\mathbb{E}[\varphi \psi]-\mathbb{E}[\varphi] \mathbb{E}[\psi]| \leq c_{m}\left|t_{1}-t_{2}\right|^{-p_{1}}\|\varphi\|_{L^{\infty}(\mathbb{P})}\|\psi\|_{L^{\infty}(\mathbb{P})}
$$

Assumption A5 (Space-mixing of the environment). Let $d \geq 3$. There exists $p_{2}>$ $2 d /(d-2)$ such that for every $m \in \mathbb{N}$ and for every $x \in \mathbb{Z}^{d}$ the following holds: For each $\varphi, \psi \in B(\Omega)$ of the form $\varphi(\omega)=\tilde{\varphi}\left(\omega\left(t_{0}\right)\right)$ and $\psi(\omega)=\tilde{\psi}\left(\omega\left(t_{0}\right)\right)$ for some $\tilde{\varphi}, \tilde{\psi} \in C_{b, \mathrm{loc}}^{1}(\hat{\Omega})$ depending on $m$ variables we have

$$
\left|\mathbb{E}\left[\varphi(\omega) \psi\left(\tau_{0, x} \omega\right)\right]-\mathbb{E}[\varphi] \mathbb{E}[\psi]\right| \leq c_{m}|x|^{-p_{2}}\|\varphi\|_{L^{\infty}(\mathbb{P})}\|\psi\|_{L^{\infty}(\mathbb{P})}
$$


We are now ready to state the following QFCLT as our main result.

Theorem 1.3. Let $d \geq 3$ and suppose that Assumptions A1-A5 hold. Then, $\mathbb{P}-a . s . X^{(\varepsilon)}$ converges (under $P_{0,0}^{\omega}$ ) in law to a Brownian motion on $\mathbb{R}^{d}$ with a deterministic nondegenerate covariance matrix $\Sigma$.

Notice that Theorem 1.3 only covers the transient lattice dimensions $d \geq 3$. In order to get an invariance principle for $X$ also in dimensions $d \leq 2$, we need to modify the mixing assumptions as follows.

Assumption A4'. Assumption A4 holds with $p_{1}>d+1$ if $d \geq 2$ and $p_{1}>4$ if $d=1$.

Assumption A5'. There exists $p_{2}>1$ such that for every $m \in \mathbb{N}$ and for every $L>0$ the following holds: For each $\varphi, \psi \in B(\Omega)$ of the form $\varphi(\omega)=\tilde{\varphi}\left(\omega\left(t_{1}\right)\right)$ and $\psi(\omega)=\tilde{\psi}\left(\omega\left(t_{2}\right)\right)$, where $\left|t_{1}-t_{2}\right| \leq L$ and $\tilde{\varphi}, \tilde{\psi} \in C_{b, \text { loc }}^{1}(\hat{\Omega})$ depend on variables contained in two subsets $A_{\varphi}$ and $A_{\psi}$ of $\mathbb{Z}^{d}$ with diameter at most $m$ and $\operatorname{dist}\left(A_{\varphi}, A_{\psi}\right) \geq L$,

$$
|\mathbb{E}[\varphi \psi]-\mathbb{E}[\varphi] \mathbb{E}[\psi]| \leq c_{m} L^{-p_{2}}\|\varphi\|_{L^{\infty}(\mathbb{P})}\|\psi\|_{L^{\infty}(\mathbb{P})}
$$

Theorem 1.4. Let $d \geq 1$ and suppose that Assumptions A1-A3, A4' and A5' hold. Then, $\mathbb{P}$-a.s. $X^{(\varepsilon)}$ converges (under $P_{0,0}^{\omega}$ ) in law to a Brownian motion on $\mathbb{R}^{d}$ with a deterministic non-degenerate covariance matrix $\Sigma$.

Remark 1.5. One can also consider the time-inhomogeneous constant speed random walk or CSRW $Y=\left(Y_{t}, t \in \mathbb{R}, P_{s, x}^{\omega},(s, x) \in \mathbb{R} \times \mathbb{Z}^{d}\right)$ with generator given by:

$$
\mathcal{L}_{t}^{Y} f(x)=\sum_{y \sim x} \frac{\mu_{x y}(t)}{\mu_{x}(t)}(f(y)-f(x)) .
$$

In contrast to the VSRW $X$, whose waiting time at any site $x \in \mathbb{Z}^{d}$ depends on $x$, the $C S R W$ waits at each site an exponential time with mean one. Since the CSRW is a time change of the VSRW, an invariance principle for $Y$ follows from an invariance principle for $X$ by the same arguments as in $[A B D H$, Section 6.2]. In this case the limiting object is a Brownian motion in $\mathbb{R}^{d}$ with covariance matrix $\Sigma_{C}=\left(1 / \mathbb{E} \mu_{0}(0)\right) \Sigma_{V}$, where $\Sigma_{V}$ denotes the covariance matrix of the limiting Brownian motion in the invariance principle for $X$.

Next we state some consequences of our results, which follow from arguments in [BH] by combining the invariance principle for $X$ and the Gaussian bound for the heat kernel. First, we have a local limit theorem for the heat kernel. Write

$$
k_{t}(x)=k_{t}^{(\Sigma)}(x)=\frac{1}{\sqrt{(2 \pi t)^{d} \operatorname{det} \Sigma}} \exp \left(-x \cdot \Sigma^{-1} x / 2 t\right)
$$

for the Gaussian heat kernel with diffusion matrix $\Sigma$.

Theorem 1.6. Let $T>0$. For $x \in \mathbb{R}^{d}$ write $\lfloor x\rfloor=\left(\left\lfloor x_{1}\right\rfloor, \ldots\left\lfloor x_{d}\right\rfloor\right)$. 
i) Suppose that Assumptions A1-A3 hold. Then,

$$
\lim _{n \rightarrow \infty} \sup _{x \in \mathbb{R}^{d}} \sup _{t \geq T}\left|n^{d / 2} \mathbb{E}\left[p^{\omega}\left(0,0 ; n t,\left\lfloor n^{1 / 2} x\right\rfloor\right)\right]-k_{t}(x)\right|=0 .
$$

ii) Under the assumptions of Theorem 1.3 or Theorem 1.4 we have

$$
\lim _{n \rightarrow \infty} \sup _{x \in \mathbb{R}^{d}} \sup _{t \geq T}\left|n^{d / 2} p^{\omega}\left(0,0 ; n t,\left\lfloor n^{1 / 2} x\right\rfloor\right)-k_{t}(x)\right|=0, \quad \mathbb{P} \text {-a.s. }
$$

Proof. Given the annealed or quenched invariance principle and the heat kernel bounds in Proposition 1.1 this can be proven as in Section 4 of [BH].

When $d \geq 3$ the calculations in Section 6 of $[\mathrm{BH}]$ then give the following bound on the Green kernel $g^{\omega}(x, y)$ defined by

$$
g^{\omega}(x, y)=\int_{0}^{\infty} p^{\omega}(0, x ; t, y) d t .
$$

Theorem 1.7. Let $d \geq 3$ and suppose that the assumptions of Theorem 1.3 or Theorem 1.4 hold.

i) There exist constants $c_{1}$ and $c_{2}$ such that for $x \neq y$

$$
\frac{c_{1}}{|x-y|^{d-2}} \leq g^{\omega}(x, y) \leq \frac{c_{2}}{|x-y|^{d-2}} .
$$

ii) Let $C=\Gamma\left(\frac{d}{2}-1\right) / 2 \pi^{d / 2} \operatorname{det} \Sigma$. For any $\varepsilon>0$ there exists $M=M(\varepsilon, \omega)$ with $\mathbb{P}[M<\infty]=1$ such that

$$
\frac{(1-\varepsilon) C}{|x|^{d-2}} \leq g^{\omega}(0, x) \leq \frac{(1+\varepsilon) C}{|x|^{d-2}} \quad \text { for }|x|>M(\omega) .
$$

iii) We have, $\mathbb{P}$-a.s.,

$$
\lim _{|x| \rightarrow \infty}|x|^{2-d} g^{\omega}(0, x)=\lim _{|x| \rightarrow \infty}|x|^{2-d} \mathbb{E}\left[g^{\omega}(0, x)\right]=C .
$$

In the case of static conductances, quenched invariance principles for the random conductance model have been proven by a number of different authors under various restrictions on the law of the conductances, see [SS, BP, Ma, BD]. Recently, these results have been unified in $[\mathrm{ABDH}]$, where a QFCLT has been obtained for the RCM with general nonnegative i.i.d. conductances. We also refer the reader to [Bi] for a recent survey on this topic.

On the other hand, to our knowledge the present paper is the first one proving an invariance principle for the RCM with a time-dynamic environment. However, quenched invariance principles have been proven for several other discrete-time random walks in 
a dynamic random environment. In [BMP1] a QFCLT is obtained for random walks in space-time product environments by using Fourier-analytic methods. This result has been improved in [BMP2] to environments satisfying an exponential spatial mixing assumption and in [BZ] to Markovian environments by using more probabilistic techniques. Another very successful approach is the well-established Kipnis-Varadhan technique based on the process of the environment as seen from the particle. In [RS1] this approach has been used to get a QFCLT for the random walk in space-time product environments. Moreover, it has been applied in [DoLi] to random walks in a dynamic enviroment, which forms a Gibbsian Markov chain in time with spatial mixing, and in [JR] to random walks on $\mathbb{R}^{d}$, where the environment is i.i.d. in time and polynomially mixing in space. Recently, a general class of random walks in an ergodic Markovian environment satisfying some coupling conditions has been studied in [RV].

Also in this paper we will follow the approach in [RS1], so we use the process of the environment as seen from the particle and the method of the 'corrector', that is we decompose the random walk $X$ into a martingale and a time-dependent corrector function. Due to the time-inhomogeneity and the resulting lack of reversibility we need to apply the adaptions of the Kipnis-Varadhan method to non-reversible situations in [MW] and [KLO]. In particular, in order to construct the corrector we show that the generator of the environment seen from the particle is a perturbation of a normal operator in the sense of [KLO, Section 2.7.5]. This is done in Section 2. As a byproduct this will already imply the annealed FCLT in Theorem 1.2.

Once the corrector is constructed, the QFCLT for the martingale part is standard, so it remains to control the corrector. To that aim we still follow [RS1] and apply the theory of 'fractional coboundaries' of Derriennic and Lin in [DeLi]. The main step in this approach is to establish a subdiffusive bound on the corrector (see Proposition 3.1 below), which is done in Section 3. To obtain this bound we establish so-called two-walk estimates, i.e. we consider the difference of two independent copies of $X$ evolving in the same fixed environment $\omega$ (cf. e.g. [JR] or Appendix A in [RS2]). In $d \geq 3$, following [Mou] we show that the variance decay of the environment viewed from the particle is strong enough for our purposes by using the mixing assumption A4 and A5 (see Lemma 3.3). In the recurrent lattice dimensions $d \leq 2$ the estimate for the variance decay is not good enough, so we give a different argument here involving the modified mixing asumptions A4' and A5'.

In Section 4 we prove the main result, i.e. we state a tightness result, which is a direct consequence from the heat kernel bounds in Proposition 1.1, and show the QFCLT for the martingale part. To control the corrector we apply the results in [DeLi], which are stated in the discrete-time setting. Since it is not clear to us, how to apply them directly in the continuous-time setting, we first prove the QFCLT for the discretized process as in [BD]. More precisely, we define $\widehat{X}_{n}=X_{n}, n \in \mathbb{N}$, and consider the process

$$
\widehat{X}_{t}^{(\varepsilon)}=\varepsilon \widehat{X}_{\left\lfloor t / \varepsilon^{2}\right\rfloor}
$$

We can control $\sup _{t \leq T}\left|X_{t}^{(\varepsilon)}-\widehat{X}_{t}^{(\varepsilon)}\right|$ - see Lemma 4.2 - so an invariance principle for $X^{(\varepsilon)}$ will follow from one for $\widehat{X}^{(\varepsilon)}$. 
Finally, in Section 5 we point out a link to stochastic interface models (see [F]). Namely, a local limit theorem for the RCM with dynamic conductances can be used to obtain scaling limits for the space-time covariation of the Ginzburg-Landau interface model via Helffer-Sjöstrand representation.

Throughout the paper we write $c$ to denote a positive constant which may change on each appearance. Constants denoted $c_{i}$ will be the same through each argument.

Acknowledgement. I thank Martin Barlow, Jean-Dominique Deuschel and Martin Slowik for helpful discussions and useful comments.

\section{Construction of the Corrector}

Throughout this section we suppose that Assumptions A1-A3 hold. We define the process of the environment seen from the particle by

$$
\eta_{t}(\omega)=\tau_{t, X_{t}} \omega, \quad \omega \in \Omega, t \geq 0 .
$$

Proposition 2.1. $\quad$ i) The process $\left(\eta_{t}\right)_{t \geq 0}$ is Markovian with transition semigroup

$$
P_{t} f(\omega)=\sum_{y \in \mathbb{Z}^{d}} p^{\omega}(0,0 ; t, y) f\left(\tau_{t, y} \omega\right) \quad \text { for all } f \in B(\Omega)
$$

The semigroup $\left(P_{t}\right)$ extends uniquely to a strongly continuous semigroup of contractions on $L^{2}(\mathbb{P})$, whose generator $L: \mathcal{D}(L) \rightarrow L^{2}(\mathbb{P})$ is given by

$$
L f(\omega)=D_{t} f(\omega)+\sum_{y \sim 0} \mu_{0 y}^{\omega}(0)\left(f\left(\tau_{0, y} \omega\right)-f(\omega)\right)
$$

with domain $\mathcal{D}(L)=\mathcal{D}\left(D_{t}\right)$.

ii) The measure $\mathbb{P}$ is invariant and ergodic for $\left(\eta_{t}\right)$.

Proof. i) The Markov property as well as the representation of the semigroup follow from (1.3) by similar arguments as in Lemma 3.1 in [KLO]. For every bounded $f \in \mathcal{D}\left(D_{t}\right)$ we have

$\frac{P_{t} f(\omega)-f(\omega)}{t}=\sum_{y \in \mathbb{Z}^{d}} \frac{p^{\omega}(0,0 ; t, y)}{t}\left(f\left(\tau_{0, y} \omega\right)-f(\omega)\right)+\sum_{y \in \mathbb{Z}^{d}} p^{\omega}(0,0 ; t, y) \frac{f\left(\tau_{t, y} \omega\right)-f\left(\tau_{0, y} \omega\right)}{t}$.

Taking limits for $t \downarrow 0$, using the fact that $p^{\omega}(0,0 ; t, y) \rightarrow \delta_{0 y}$, we obtain the formula for $L f$. Obviously, the operators $L$ and $D_{t}$ have the same domain.

ii) Let $f \in \mathcal{D}(L)$. Since the operator $D_{t}$ is anti-selfadjoint we have $\left\langle D_{t} f\right\rangle_{\mathbb{P}}=0$. Hence,

$$
\begin{aligned}
\langle L f\rangle_{\mathbb{P}} & =\sum_{y \in \mathbb{Z}^{d}}\left\langle\mu_{0 y}^{\omega}(0) f\left(\tau_{0, y} \omega\right)\right\rangle_{\mathbb{P}}-\left\langle\mu_{0 y}^{\omega}(0) f(\omega)\right\rangle_{\mathbb{P}}=\sum_{y \in \mathbb{Z}^{d}}\left\langle\mu_{0 y}^{\tau_{0,-y} \omega}(0) f(\omega)\right\rangle_{\mathbb{P}}-\left\langle\mu_{0 y}^{\omega}(0) f(\omega)\right\rangle_{\mathbb{P}} \\
& =\sum_{y \in \mathbb{Z}^{d}}\left\langle\mu_{0,-y}^{\omega}(0) f(\omega)\right\rangle_{\mathbb{P}}-\left\langle\mu_{0 y}^{\omega}(0) f(\omega)\right\rangle_{\mathbb{P}}=0
\end{aligned}
$$


where we have used the invariance of $\mathbb{P}$ w.r.t. $\tau_{t, x}$ and (1.3). Thus, $\mathbb{P}$ is an invariant measure for $\eta$. To prove that $\mathbb{P}$ is also ergodic, let now $A \in \mathcal{F}$ with $P_{t} \mathbb{1}_{A}=\mathbb{1}_{A}$. Then,

$$
0=\mathbb{1}_{A^{c}}(\omega) \cdot P_{t} \mathbb{1}_{A}(\omega)=\sum_{y \in \mathbb{Z}^{d}} \mathbb{1}_{A^{c}}(\omega) p^{\omega}(0,0 ; t, y) \mathbb{1}_{A}\left(\tau_{t, y} \omega\right)
$$

Since for all $t>0$ and $y \in \mathbb{Z}^{d}$ there is a stricly positive lower bound for $p^{\omega}(0,0 ; t, y)$ independent of $\omega$ (see Proposition 4.3 in [DD]) we get

$$
\mathbb{1}_{A^{c}}(\omega) \cdot \mathbb{1}_{A}\left(\tau_{t, y} \omega\right)=0
$$

Thus, the set $A$ is invariant under $\tau_{t, x}$. Since $\mathbb{P}$ is ergodic w.r.t. $\tau_{t, x}$ we conclude that $A$ is $\mathbb{P}$-trivial and the claim follows.

Lemma 2.2. For $f \in \mathcal{D}(L)$,

$$
\langle f,(-L) f\rangle_{\mathbb{P}}=\frac{1}{2} \sum_{y \in \mathbb{Z}^{d}} \mathbb{E}\left[\mu_{0 y}^{\omega}(0)\left(f\left(\tau_{0, y} \omega\right)-f(\omega)\right)^{2}\right] .
$$

Proof. Recall that $\left\langle f, D_{t} f\right\rangle_{\mathbb{P}}=0$. Therefore,

$$
\begin{aligned}
& \langle f,(-L) f\rangle_{\mathbb{P}}=-\sum_{y \in \mathbb{Z}^{d}} \mathbb{E}\left[f(\omega) \mu_{0 y}^{\omega}(0)\left(f\left(\tau_{0, y} \omega\right)-f(\omega)\right)\right] \\
= & -\frac{1}{2} \sum_{y \in \mathbb{Z}^{d}} \mathbb{E}\left[f(\omega) \mu_{0 y}^{\omega}(0)\left(f\left(\tau_{0, y} \omega\right)-f(\omega)\right)\right]-\frac{1}{2} \sum_{y \in \mathbb{Z}^{d}} \mathbb{E}\left[f(\omega) \mu_{0,-y}^{\omega}(0)\left(f\left(\tau_{0,-y} \omega\right)-f(\omega)\right)\right] \\
= & -\frac{1}{2} \sum_{y \in \mathbb{Z}^{d}} \mathbb{E}\left[f(\omega) \mu_{0 y}^{\omega}(0)\left(f\left(\tau_{0, y} \omega\right)-f(\omega)\right)\right]-\frac{1}{2} \sum_{y \in \mathbb{Z}^{d}} \mathbb{E}\left[f\left(\tau_{0, y} \omega\right) \mu_{0,-y}^{\tau_{0, y} \omega}(0)\left(f(\omega)-f\left(\tau_{0, y} \omega\right)\right)\right] \\
= & \frac{1}{2} \sum_{y \in \mathbb{Z}^{d}} \mathbb{E}\left[\mu_{0 y}^{\omega}(0)\left(f\left(\tau_{0, y} \omega\right)-f(\omega)\right)^{2}\right]
\end{aligned}
$$

where we have used again the invariance of $\mathbb{P}$ w.r.t. $\tau_{t, x}$ and (1.3).

Let $P_{t}^{*}$ and $L^{*}$ denote the $L^{2}(\mathbb{P})$-adjoint operators of $P_{t}$ and $L$, respectively.

Proposition 2.3. We have

$$
P_{t}^{*} f(\omega)=\sum_{y \in \mathbb{Z}^{d}} \hat{p}^{\omega}(0,0 ; t, y) f\left(\tau_{-t, y} \omega\right), \quad f \in L^{2}(\mathbb{P})
$$

with $\hat{p}^{\omega}(s, x ; t, y):=p^{\omega}(-t, y ;-s, x)$ and for $f \in \mathcal{D}(L)$

$$
L^{*} f(\omega)=-D_{t} f(\omega)+\sum_{y \sim 0} \mu_{0 y}^{\omega}(0)\left(f\left(\tau_{0, y} \omega\right)-f(\omega)\right) .
$$


Proof. Using (1.3) we compute the adjoint of $P_{t}$ as

$$
\begin{aligned}
\left\langle P_{t} f, g\right\rangle_{\mathbb{P}} & =\sum_{y \in \mathbb{Z}^{d}} \mathbb{E}\left[p^{\omega}(0,0 ; t, y) f\left(\tau_{t, y} \omega\right) g(\omega)\right]=\sum_{y \in \mathbb{Z}^{d}} \mathbb{E}\left[p^{\tau_{-t,-y} \omega}(0,0 ; t, y) f(\omega) g\left(\tau_{-t,-y} \omega\right)\right] \\
& =\sum_{y \in \mathbb{Z}^{d}} \mathbb{E}\left[p^{\omega}(-t,-y ; 0,0) f(\omega) g\left(\tau_{-t,-y} \omega\right)\right]=\sum_{y \in \mathbb{Z}^{d}} \mathbb{E}\left[\hat{p}^{\omega}(0,0 ; t, y) g\left(\tau_{-t, y} \omega\right) f(\omega)\right]
\end{aligned}
$$

and the representation for $P_{t}^{*}$ follows. To compute $L^{*}$ we use a similar procedure as in Lemma 2.2 and get

$$
\begin{aligned}
\langle L f, g\rangle_{\mathbb{P}} & =\left\langle D_{t} f, g\right\rangle_{\mathbb{P}}+\sum_{y \in \mathbb{Z}^{d}} \mathbb{E}\left[\mu_{0 y}^{\omega}(0)\left(f\left(\tau_{0, y} \omega\right)-f(\omega)\right) g(\omega)\right] \\
& =-\left\langle f, D_{t} g\right\rangle_{\mathbb{P}}-\frac{1}{2} \sum_{y \in \mathbb{Z}^{d}} \mathbb{E}\left[\mu_{0 y}^{\omega}(0)\left(f\left(\tau_{0, y} \omega\right)-f(\omega)\right)\left(g\left(\tau_{0, y} \omega\right)-g(\omega)\right)\right] \\
& =-\left\langle f, D_{t} g\right\rangle_{\mathbb{P}}+\sum_{y \in \mathbb{Z}^{d}} \mathbb{E}\left[\mu_{0 y}^{\omega}(0)\left(g\left(\tau_{0, y} \omega\right)-g(\omega)\right) f(\omega)\right],
\end{aligned}
$$

which gives the claim.

Next we introduce the Hilbert spaces $\mathcal{H}_{1}$ and $\mathcal{H}_{-1}$. Let $\mathcal{C}$ be a common core of the operators $L$ and $L^{*}$. On $\mathcal{C}$ we define the seminorm

$$
\|f\|_{\mathcal{H}_{1}}^{2}=\langle f,(-L) f\rangle_{\mathbb{P}}, \quad f \in \mathcal{C} .
$$

Let $\mathcal{H}_{1}$ be the completion of $\mathcal{C}$ (or more precisely the completion of equivalence classes of elements in $\mathcal{C}$ w.r.t. the equivalence relation $f \sim g$ if $\|f-g\|_{\mathcal{H}_{1}}=0$ ) w.r.t. $\|\cdot\|_{\mathcal{H}_{1}}$. Then, $\mathcal{H}_{1}$ is a Hilbert space with inner product $\langle., .\rangle_{\mathcal{H}_{1}}$ given by polarization:

$$
\langle f, g\rangle_{\mathcal{H}_{1}}=\frac{1}{4}\left(\|f+g\|_{\mathcal{H}_{1}}^{2}-\|f-g\|_{\mathcal{H}_{1}}^{2}\right)
$$

Associated to $\mathcal{H}_{1}$ we define the dual space $\mathcal{H}_{-1}$ as follows. For $f \in L^{2}(\mathbb{P})$ let

$$
\|f\|_{\mathcal{H}_{-1}}^{2}=\sup _{g \in \mathcal{C}}\left(2\langle f, g\rangle_{\mathbb{P}}-\|g\|_{\mathcal{H}_{1}}^{2}\right) \text {. }
$$

The Hilbert space $\mathcal{H}_{-1}$ is then defined as the $\|\cdot\|_{\mathcal{H}_{-1}}$-completion of (equivalence classes of) elements in $\mathcal{C}$ with finite $\|\cdot\|_{\mathcal{H}_{-1}}$-norm. As before the inner product $\langle., .\rangle_{\mathcal{H}_{-1}}$ is defined through polarization. We refer to Section 2.2 in [KLO] for more details.

Next we define the local drift

$$
V_{j}(\omega)=\sum_{y \sim 0} \mu_{0 y}^{\omega}(0) y^{j}=\mathcal{L}_{0}^{\omega} f_{j}(0), \quad j=1, \ldots, d
$$

where $f_{j}(x)=x^{j}, x^{j}$ and $y^{j}$ denoting the $j$-th component of $x$ and $y$. Since $\mu_{0 y}(0)=0$ unless $y \sim 0$, we have $V_{j}(\omega)=\mu_{0, e_{j}}^{\omega}(0)-\mu_{0,-e_{j}}^{\omega}$. 
Lemma 2.4. For every $j=1, \ldots, d, V_{j} \in L^{2}(\mathbb{P}) \cap \mathcal{H}_{-1}$.

Proof. It suffices to show that

$$
\left|\left\langle V_{j}, f\right\rangle_{\mathbb{P}}\right|^{2} \leq c\langle f,(-L) f\rangle_{\mathbb{P}} \quad \text { for all } f \in \mathcal{H}_{1}
$$

(cf. equation (2.12) in [KLO])). By definition of $V_{j}$ we have

$$
\begin{aligned}
\left\langle V_{j}, f\right\rangle_{\mathbb{P}} & =\mathbb{E}\left[\mu_{0, e_{j}}^{\omega}(0) f(\omega)\right]-\mathbb{E}\left[\mu_{0,-e_{j}}^{\omega}(0) f(\omega)\right]=\mathbb{E}\left[\mu_{0, e_{j}}^{\omega}(0) f(\omega)\right]-\mathbb{E}\left[\mu_{0, e_{j}}^{\omega}(0) f\left(\tau_{0, e_{j}} \omega\right)\right] \\
& =-\mathbb{E}\left[\mu_{0, e_{j}}^{\omega}(0)\left(f\left(\tau_{0, e_{j}} \omega\right)-f(\omega)\right)\right] .
\end{aligned}
$$

Hence, using Cauchy Schwarz and Lemma 2.2

$$
\begin{aligned}
\left|\left\langle V_{j}, f\right\rangle_{\mathbb{P}}\right|^{2} & \leq \mathbb{E}\left[\mu_{0, e_{j}}^{\omega}(0)\right] \mathbb{E}\left[\mu_{0, e_{j}}^{\omega}(0)\left(f\left(\tau_{0, e_{j}} \omega\right)-f(\omega)\right)^{2}\right] \leq C_{u} \sum_{y \in \mathbb{Z}^{d}} \mathbb{E}\left[\mu_{0 y}^{\omega}(0)\left(f\left(\tau_{0, y} \omega\right)-f(\omega)\right)^{2}\right] \\
& =2 C_{u}\langle f,(-L) f\rangle_{\mathbb{P}},
\end{aligned}
$$

and we obtain (2.1).

For $\lambda>0$, we consider for each $j$ the solution $u_{\lambda}^{j}$ of the resolvent equation

$$
(\lambda-L) u_{\lambda}^{j}=V_{j} .
$$

Proposition 2.5. For every $j=1, \ldots, d$, there exists $u^{j} \in \mathcal{H}_{1}$ such that

$$
\lim _{\lambda \rightarrow 0} \lambda\left\|u_{\lambda}^{j}\right\|_{L^{2}(\mathbb{P})}^{2}=0 \quad \text { and } \quad \lim _{\lambda \rightarrow 0} u_{\lambda}^{j}=u^{j} \text { strongly in } \mathcal{H}_{1} .
$$

The proof of Proposition 2.5 will be based on the following statement proven in [KLO].

Proposition 2.6. Suppose we have the decomposition $L=L^{0}+B$ of the operator $L$ such that

i) The operator $L^{0}$ is normal, i.e. $L^{0}\left(L^{0}\right)^{*}=\left(L^{0}\right)^{*} L^{0}$.

ii) The Dirichlet forms of $L$ and $L^{0}$ are equivalent, i.e. there exist positive constants $c_{1}$ and $c_{2}$ such that

$$
c_{1}\langle f,(-L) f\rangle_{\mathbb{P}} \leq\left\langle f,\left(-L^{0}\right) f\right\rangle_{\mathbb{P}} \leq c_{2}\langle f,(-L) f\rangle_{\mathbb{P}}, \quad \text { for all } f \in \mathcal{D}(L) .
$$

iii) B satisfies a sector condition w.r.t. $L^{0}$, i.e. there exists a positive constant $c$ such that

$$
\langle f, B g\rangle_{\mathbb{P}}^{2} \leq c\left\langle f,\left(-L^{0}\right) f\right\rangle_{\mathbb{P}}\left\langle g,\left(-L^{0}\right) g\right\rangle_{\mathbb{P}}, \quad f, g \in \mathcal{D}(L) .
$$

Then, for any fixed $V \in L^{2}(\mathbb{P}) \cap \mathcal{H}_{-1}$ the solution $f_{\lambda}$ of the resolvent equation $(\lambda-L) f_{\lambda}=V$ satisfies

$$
\lim _{\lambda \rightarrow 0} \lambda\left\|f_{\lambda}\right\|_{L^{2}(\mathbb{P})}^{2}=0 \quad \text { and } \quad \lim _{\lambda \rightarrow 0} f_{\lambda}=\text { fstrongly in } \mathcal{H}_{1},
$$

for some $f \in \mathcal{H}_{1}$. 
Proof. By Proposition 2.25 in [KLO] the assumptions imply that

$$
\sup _{0<\lambda \leq 1}\left\|L f_{\lambda}\right\|_{\mathcal{H}_{-1}}<\infty .
$$

The claim follows then from Lemma 2.16 in [KLO].

Proof of Proposition 2.5. We decompose the operator $L=L^{0}+B$ with

$$
L^{0} f:=D_{t} f+\sum_{y \sim 0} C_{l}\left(f\left(\tau_{0, y} \omega\right)-f(\omega)\right), \quad f \in \mathcal{D}(L),
$$

and

$$
B f:=\sum_{y \sim 0}\left(\mu_{0 y}^{\omega}(0)-C_{l}\right)\left(f\left(\tau_{0, y} \omega\right)-f(\omega)\right), \quad f \in \mathcal{D}(L) .
$$

A similar calculation as in the proof of Lemma 2.2 shows that

$$
\begin{aligned}
\left\langle f,\left(-L^{0}\right) f\right\rangle_{\mathbb{P}} & =\frac{1}{2} \sum_{y \sim 0} \mathbb{E}\left[C_{l}\left(f\left(\tau_{0, y} \omega\right)-f(\omega)\right)^{2}\right] \\
\langle f,(-B) g\rangle_{\mathbb{P}} & =\frac{1}{2} \sum_{y \sim 0} \mathbb{E}\left[\left(\mu_{0 y}^{\omega}(0)-C_{l}\right)\left(f\left(\tau_{0, y} \omega\right)-f(\omega)\right)\left(g\left(\tau_{0, y} \omega\right)-g(\omega)\right)\right] .
\end{aligned}
$$

The claim will follow from Proposition 2.6 and Lemma 2.4 once we have verified conditions i)-iii) in Proposition 2.6. To show i), note that the closure of $L^{0}$ is the generator of a semigroup $\left(P_{t}^{0}\right)$ that corresponds to a process seen from the particle associated with a simple random walk on $\mathbb{Z}^{d}$ with constant jump rates $C_{l}$. In particular, the associated process is time-homogeneous, i.e. the corresponding transition probabilities satisfy $p_{0}^{\omega}(s, x ; t, y)=p_{0}^{\omega}(t-s, x, y)$ and $\hat{p}_{0}^{\omega}(s, x ; t, y)=\hat{p}_{0}^{\omega}(t-s, x, y)$, where $p_{0}^{\omega}(t, x, y)=p_{0}^{\omega}(0, x ; t, y)$ and $\hat{p}_{0}^{\omega}(t, x, y)=\hat{p}_{0}^{\omega}(0, x ; t, y)$. Since this random walk is obviously reversible w.r.t. the counting measure, we have $p_{0}^{\omega}(t, x, y)=\hat{p}_{0}^{\omega}(t, x, y)$. Then, since we have similar representations for $P_{t}^{0}$ and $\left(P_{t}^{0}\right)^{*}$ as for the semigroups in Proposition 2.1 and Proposition 2.3, we get

$$
\left(P_{t}^{0}\right)^{*} P_{t}^{0}=P_{t}^{0}\left(P_{t}^{0}\right)^{*}, \quad t \geq 0,
$$

which implies that the closure of $L^{0}$ is normal (see Theorem 13.37 in $[\mathrm{Ru}]$ ).

Condition ii) is immediate from Lemma 2.2, (2.3) and the ellipticity condition (1.5). To prove iii) we use (2.4), Cauchy Schwarz and the ellipticity condition (1.5), which gives

$$
\begin{aligned}
\langle f, B g\rangle_{\mathbb{P}}^{2} & \leq \frac{1}{2} C_{u}^{2} d \mathbb{E}\left[\sum_{y \sim 0}\left(f\left(\tau_{0, y} \omega\right)-f(\omega)\right)^{2}\right] \times \mathbb{E}\left[\sum_{y \sim 0}\left(g\left(\tau_{0, y} \omega\right)-g(\omega)\right)^{2}\right] \\
& \leq \frac{C_{u}^{2} d}{2 C_{l}^{2}}\left\langle f,\left(-L^{0}\right) f\right\rangle_{\mathbb{P}}\left\langle g,\left(-L^{0}\right) g\right\rangle_{\mathbb{P}}
\end{aligned}
$$

and the claim follows.

For abbreviation we write $u_{\lambda}=\left(u_{\lambda}^{1}, \ldots, u_{\lambda}^{d}\right)$ and

$$
\chi_{\lambda}(t, x, \omega):=u_{\lambda} \circ \tau_{t, x}-u_{\lambda} .
$$


Proposition 2.7. For all non-negative $t \in \mathbb{Q}$ and $x \in \mathbb{Z}^{d}$ the limit

$$
\lim _{\lambda^{\prime} \rightarrow 0} \chi_{\lambda^{\prime}}(t, x, \omega)=: \chi(t, x, \omega)
$$

exists along a subfamily $\left(\lambda^{\prime}\right)$ for $\mathbb{P}$-a.e. $\omega$. Moreover, the mapping $t \mapsto \chi\left(t, X_{t}, \omega\right)$ can be extended to a right-continuous function on $[0, \infty)$ such that

$$
M_{t}=X_{t}+\chi\left(t, X_{t}, \omega\right), \quad t \geq 0,
$$

is a $P_{0,0}^{\omega}$-martingale.

Proof. For every $j=1, \ldots, d$ and every $\lambda>0$ we have that for $\mathbb{P}$-a.e. $\omega$ the processes

$$
N_{t}^{j, \lambda}=u_{\lambda}^{j}\left(\eta_{t}\right)-u_{\lambda}^{j}(\omega)-\int_{0}^{t} L u_{\lambda}^{j}\left(\eta_{s}\right) d s
$$

and

$$
\tilde{M}_{t}^{j}=X_{t}^{j}-\int_{0}^{t} \mathcal{L}_{s}^{\omega} f_{j}\left(X_{s}\right) d s
$$

are both $P_{0,0}^{\omega}$-martingales, where as before $f_{j}(x)=x^{j}$. Then, using the definition of $V_{j}$ and the fact that $u_{\lambda}^{j}$ solves the resolvent equation (2.2) we get

$$
\begin{aligned}
X_{t}^{j} & =\tilde{M}_{t}^{j}+\int_{0}^{t} \mathcal{L}_{s}^{\omega} f_{j}\left(X_{s}\right) d s=\tilde{M}_{t}^{j}+\int_{0}^{t} V_{j}\left(\eta_{s}\right) d s=\tilde{M}_{t}^{j}+\int_{0}^{t}(\lambda-L) u_{\lambda}^{j}\left(\eta_{s}\right) d s \\
& =\tilde{M}_{t}^{j}+N_{t}^{j, \lambda}-\left(u_{\lambda}^{j}\left(\eta_{t}\right)-u_{\lambda}^{j}(\omega)\right)+\lambda \int_{0}^{t} u_{\lambda}^{j}\left(\eta_{s}\right) d s .
\end{aligned}
$$

In a first step we show that the martingale $N_{t}^{j, \lambda}$ converges in $L^{2}\left(\mathbb{P} \otimes P_{0,0}^{\omega}\right)$ as $\lambda \downarrow 0$ to a martingale $N_{t}^{j}$. To that aim it is enough to prove that $N_{t}^{j, \lambda}$ is a Cauchy sequence in $L^{2}\left(\mathbb{P} \otimes P_{0,0}^{\omega}\right)$. Since $\mathbb{P}$ is an invariant measure for $\eta$ we use Lemma 2.2 to obtain

$$
\begin{aligned}
\mathbb{E} E_{0,0}^{\omega}\left\langle N^{j, \lambda}-N^{j, \lambda^{\prime}}\right\rangle_{t} & =\int_{0}^{t} \mathbb{E} E_{0,0}^{\omega}\left[L\left(u_{\lambda}^{j}-u_{\lambda^{\prime}}^{j}\right)^{2}-2\left(u_{\lambda}^{j}-u_{\lambda^{\prime}}^{j}\right) L\left(u_{\lambda}^{j}-u_{\lambda^{\prime}}^{j}\right)\right]\left(\eta_{s}\right) d s \\
& =2 t\left\langle\left(u_{\lambda}^{j}-u_{\lambda^{\prime}}^{j}\right),(-L)\left(u_{\lambda}^{j}-u_{\lambda^{\prime}}^{j}\right)\right\rangle_{\mathbb{P}} \\
& =2 t\left\|u_{\lambda}^{j}-u_{\lambda^{\prime}}^{j}\right\|_{\mathcal{H}_{1}}^{2},
\end{aligned}
$$

which implies that $N_{t}^{j, \lambda}$ is a Cauchy sequence in $L^{2}\left(\mathbb{P} \otimes P_{0,0}^{\omega}\right)$ by Proposition 2.5. Thus, the martingale $M_{t}^{j, \lambda}=\tilde{M}_{t}^{j}+N_{t}^{j, \lambda}$ converges to a martingale, whose right-continuous modification we denote by $M_{t}^{j}$. We define $M_{t}=\left(M_{t}^{1}, \ldots, M_{t}^{d}\right)$.

The next step is to show that the last term in (2.9) converges to zero in $L^{2}\left(\mathbb{P} \otimes P_{0,0}^{\omega}\right)$ as $\lambda \downarrow 0$. Since $V_{j} \in \mathcal{H}_{-1}$ we have that $\lim _{\lambda} \lambda u_{\lambda}^{j}=0$ in $L^{2}(\mathbb{P})$ (cf. equation (2.15) in [KLO]). Thus, for every $j=1, \ldots, d$,

$$
\left\|\lambda \int_{0}^{t} u_{\lambda}^{j}\left(\eta_{s}\right) d s\right\|_{L^{2}\left(\mathbb{P} \otimes P_{0,0}^{\omega}\right)} \leq \lambda \int_{0}^{t}\left\|u_{\lambda}^{j}\left(\eta_{s}\right)\right\|_{L^{2}\left(\mathbb{P} \otimes P_{0,0}^{\omega}\right)} d s=t \lambda\left\|u_{\lambda}^{j}\right\|_{L^{2}(\mathbb{P})} \rightarrow 0 .
$$


Thus, by taking $L^{2}\left(\mathbb{P} \otimes P_{0,0}^{\omega}\right)$-limits in $(2.9)$ we get that $\chi_{\lambda}\left(t, X_{t}, \cdot\right)$ converges in $L^{2}(\mathbb{P} \otimes$ $\left.P_{0,0}^{\omega}\right)$ as $\lambda \downarrow 0$ for every $t \geq 0$. By a diagonal procedure we can extract a suitable subsequence $\lambda^{\prime}$ such that for $\mathbb{P}$-a.e. $\omega$ we have that $\chi_{\lambda}\left(t, X_{t}, \omega\right)$ has a limit in $L^{2}\left(P_{0,0}^{\omega}\right)$ and $P_{0,0}^{\omega}$-a.s. along $\lambda^{\prime}$ for all non-negative $t \in \mathbb{Q}$. In particular, the limit is $\sigma\left(X_{t}\right)$-measurable and will therefore be denoted by $\chi\left(t, X_{t}, \omega\right)$. Hence,

$$
X_{t}=M_{t}+\chi\left(t, X_{t}, \omega\right)
$$

Moreover, for $\mathbb{P}$-a.e. $\omega$,

$$
\sum_{y \in \mathbb{Z}^{d}} p^{\omega}(0,0 ; t, y)\left|\chi_{\lambda}(t, y, \omega)-\chi(t, y, \omega)\right|^{2}=E_{\omega}^{0}\left|\left(\chi_{\lambda}\left(t, X_{t}, \omega\right)\right)-\chi\left(t, X_{t}, \omega\right)\right|^{2} \rightarrow 0
$$

along $\lambda^{\prime}$. Since $p^{\omega}(0,0 ; t, y)>0$ for all $t>0$ and $x \in \mathbb{Z}^{d}$, we conclude that for $\mathbb{P}$-a.e. $\omega$ the limit in (2.5) exists for every non-negative $t \in \mathbb{Q}$ and every $y \in \mathbb{Z}^{d}$. Finally, using (2.10) and the fact that $X_{t}$ and $M_{t}$ have right-continuous trajectories, we can extend $\chi\left(t, X_{t}, \omega\right)$ to a right-continuous function on $[0, \infty)$ and $(2.6)$ follows.

Remark 2.8. Note that for all non-negative $s, t \in \mathbb{Q}$ and $x, y \in \mathbb{Z}^{d}$,

$$
\begin{aligned}
u_{\lambda}\left(\tau_{t, y} \omega\right)-u_{\lambda}\left(\tau_{s, x} \omega\right) & =\left(u_{\lambda}\left(\tau_{t, y} \omega\right)-u_{\lambda}(\omega)\right)-\left(u_{\lambda}\left(\tau_{s, x} \omega\right)-u_{\lambda}(\omega)\right) \\
& \rightarrow \chi(t, y, \omega)-\chi(s, x, \omega)
\end{aligned}
$$

along the chosen subsequence for $\mathbb{P}$-a.e. $\omega$. The function $h_{\lambda}\left(\omega_{0}, \omega_{1}\right):=u_{\lambda}\left(\omega_{1}\right)-u_{\lambda}\left(\omega_{0}\right)$ on $\Omega \times \Omega$ converges in $L^{2}\left(\Omega \times \Omega, \mathbb{P} \circ\left(\tau_{s, x}, \tau_{t, y}\right)^{-1}\right)$ to a function $h$. In particular, for $\mathbb{P}$-a.e. $\omega$,

$$
h\left(\tau_{s, x} \omega, \tau_{t, y} \omega\right)=\chi(t, y, \omega)-\chi(s, x, \omega) .
$$

Corollary 2.9. For $\mathbb{P}$-a.e. $\omega$ the corrector satisfies the cocycle property

$$
\chi(s+t, x+y, \omega)=\chi(s, x, \omega)+\chi\left(t, y, \tau_{s, x} \omega\right)
$$

for non-negative $s, t \in \mathbb{Q}$.

Proof. We have

$$
u_{\lambda} \circ \tau_{s+t, x+y}-u_{\lambda}=\left(u_{\lambda} \circ \tau_{s, x}-u_{\lambda}\right)+\left(u_{\lambda} \circ \tau_{t, y}-u_{\lambda}\right) \circ \tau_{s, x},
$$

and the claim follows by taking the $L^{2}(\mathbb{P})$-limit along $\lambda^{\prime}$ on both sides.

In the following, for any $G: \mathbb{Z}^{d} \times \Omega \rightarrow \mathbb{R}$ we shall write

$$
\|G\|_{\omega}^{2}:=\sum_{y \sim 0} \mu_{0 y}^{\omega}(0) G(y, \omega)^{2}
$$


Corollary 2.10. For every $v \in \mathbb{R}^{d}$ the covariation process of the martingale $M^{v}:=v \cdot M$ is given by

$$
\langle v \cdot M\rangle_{t}=\int_{0}^{t}\|v \cdot \Phi\|_{\eta_{s}}^{2} d s
$$

where

$$
\Phi(x, \omega):=x+\chi(0, x, \omega)
$$

Proof. First we compute the covariation process of the martingale $M_{t}^{j, \lambda}$ defined as in the proof of Proposition 2.7. To that aim we define $z_{j, \lambda}(t, x, \omega):=x^{j}+u_{\lambda}^{j}\left(\tau_{t, x} \omega\right)$. Then, by adding (2.7) and (2.8) we get

$$
M_{t}^{j, \lambda}=z_{j, \lambda}\left(t, X_{t}, \omega\right)-z_{j, \lambda}(0,0, \omega)-\int_{0}^{t} \bar{L} z_{j, \lambda}\left(s, X_{s}, \omega\right) d s
$$

where

$$
\bar{L} z_{j, \lambda}(t, x, \omega):=D_{t} z_{j, \lambda}(t, x, \omega)+\sum_{y \sim x} \mu_{x y}^{\omega}(t)\left(z_{j, \lambda}(t, y, \omega)-z_{j, \lambda}(t, x, \omega)\right)
$$

In particular,

$$
\begin{aligned}
\bar{L} z_{j, \lambda}^{2}-2 z_{j, \lambda} \bar{L} z_{j, \lambda}(t, x, \omega) & =\sum_{y \in \mathbb{Z}^{d}} \mu_{x y}^{\omega}(t)\left(z_{j, \lambda}(t, y, \omega)-z_{j, \lambda}(t, x, \omega)\right)^{2} \\
& =\sum_{y \in \mathbb{Z}^{d}} \mu_{0, y-x}^{\tau_{t, x} \omega}(0)\left(y^{j}-x^{j}+\left[u_{\lambda}^{j} \circ \tau_{0, y-x}-u_{\lambda}^{j}\right] \circ \tau_{t, x}(\omega)\right)^{2} \\
& =\sum_{y \in \mathbb{Z}^{d}} \mu_{0, y}^{\tau_{t, x} \omega}(0)\left(y^{j}+\left[u_{\lambda}^{j} \circ \tau_{0, y}-u_{\lambda}^{j}\right] \circ \tau_{t, x}(\omega)\right)^{2},
\end{aligned}
$$

so that

$$
\left\langle M_{t}^{j, \lambda}\right\rangle_{t}=\int_{0}^{t} \sum_{y \in \mathbb{Z}^{d}} \mu_{0, y}^{\eta_{s}}(0)\left(y^{j}+\left[u_{\lambda}^{j} \circ \tau_{0, y}-u_{\lambda}^{j}\right]\left(\eta_{s}\right)\right)^{2} d s
$$

and by taking limits on both sides along $\lambda^{\prime}$, we obtain

$$
\left\langle M^{j}\right\rangle_{t}=\int_{0}^{t} \sum_{y \in \mathbb{Z}^{d}} \mu_{0 y}^{\eta_{s}}(0)\left[y^{j}+\chi^{j}\left(0, y, \eta_{s}\right)\right]^{2} d s=\int_{0}^{t}\left\|\Phi^{j}\right\|_{\eta_{s}}^{2} d s
$$

and for any $v \in \mathbb{R}^{d}$ a similar computation gives (2.11).

We conclude this section with a convergence result, which will imply the annealed invariance principle. Nevertheless, it will be convenient to complete the proof of Theorem 1.2 in Section 4 below. 
Proposition 2.11. We have $t^{-1 / 2} \chi\left(t, X_{t}, \omega\right) \rightarrow 0$ in $L^{2}\left(\mathbb{P}_{0,0}^{*}\right)$ as $t \rightarrow \infty$.

Proof. Consider an arbitrary fixed $j \in\{1, \ldots, d\}$. Still using the notation in the proof of Proposition 2.7 we have for every $t$ and any $\lambda>0$,

$$
\chi^{j}\left(t, X_{t}, \omega\right)=M_{t}^{j}-X_{t}^{j}=M_{t}^{j}-M_{t}^{j, \lambda}+u_{\lambda}^{j}\left(\eta_{t}\right)-u_{\lambda}^{j}(\omega)-\lambda \int_{0}^{t} u_{\lambda}^{j}\left(\eta_{s}\right) d s,
$$

and by Cauchy-Schwarz we get

$$
\left|\chi^{j}\left(t, X_{t}, \omega\right)\right|^{2} \leq 9\left|M_{t}^{j}-M_{t}^{j, \lambda}\right|^{2}+9\left|u_{\lambda}^{j}\left(\eta_{t}\right)-u_{\lambda}^{j}(\omega)\right|^{2}+9 \lambda^{2} \int_{0}^{t}\left|u_{\lambda}^{j}\left(\eta_{s}\right)\right|^{2} d s
$$

We argue similarly as in the proof of Proposition 2.7. Using the fact that $\mathbb{P}$ is an invariant measure for the environment process $\eta$ we obtain

$$
\begin{aligned}
\mathbb{E} E_{0,0}^{\omega}\left|M_{t}^{j}-M_{t}^{j, \lambda}\right|^{2} & \leq 2 t\left\|u_{\lambda}^{j}-u^{j}\right\|_{\mathcal{H}_{1}}^{2}, \\
\mathbb{E} E_{0,0}^{\omega}\left|u_{\lambda}^{j}\left(\eta_{t}\right)-u_{\lambda}^{j}(\omega)\right|^{2} & \leq 4\left\|u_{\lambda}^{j}\right\|_{L^{2}(\mathbb{P})}^{2}, \\
\mathbb{E} E_{0,0}^{\omega} \lambda^{2} \int_{0}^{t}\left|u_{\lambda}^{j}\left(\eta_{s}\right)\right|^{2} d s & \leq t \lambda^{2}\left\|u_{\lambda}^{j}\right\|_{L^{2}(\mathbb{P})}^{2} .
\end{aligned}
$$

Choosing $\lambda=t^{-1}$ the claim follows by Proposition 2.5.

\section{Subdiffusive Bound on the Corrector}

In this section we shall prove the following

Proposition 3.1. Under the assumptions of Theorem 1.3 or Theorem 1.4, there exists an $\alpha<1 / 2$ such that

$$
\mathbb{E} E_{0,0}^{\omega}\left[\left|\chi\left(n, X_{n}, \omega\right)\right|^{2}\right]=O\left(n^{2 \alpha}\right) .
$$

\subsection{Convergence of the Resolvents}

Proposition 3.2. Under the assumptions of Theorem 1.3 or Theorem 1.4, there exists an $\alpha<1 / 2$ such that for every $j=1, \ldots, d$,

$$
\left\|u_{\lambda}^{j}\right\|_{L^{2}(\mathbb{P})}=O\left(\lambda^{-\alpha}\right) .
$$

Note that while for the annealed FCLT the convergence in Proposition 2.11 is sufficient, we will need the stronger statement in Proposition 3.1 for the QFCLT. This difference also appears in the corresponding results on the resolvents $u_{\lambda}$, (cf. Proposition 2.5 and Proposition 3.2). Before we prove Proposition 3.2 we will first show how it implies Proposition 3.1 .

Proof of Proposition 3.1. Similarly to the proof of Proposition 2.11 we show that for a certain $\lambda$ chosen below depending on $n$ the terms in the right hand side of (2.13)-(2.15) 
are in $O\left(n^{2 \alpha}\right)$. We shall use similar arguments as in [MW], in particular cf. Lemma 2 and Corollary 4 in $[\mathrm{MW}]$. In a first step we will show that

$$
\left\|u_{\lambda}^{j}-u_{\lambda^{\prime}}^{j}\right\|_{\mathcal{H}_{1}}^{2} \leq \frac{\left(\sqrt{\lambda}+\sqrt{\lambda^{\prime}}\right)^{2}}{2}\left(\left\|u_{\lambda}^{j}\right\|_{L^{2}(\mathbb{P})}^{2}+\left\|u_{\lambda^{\prime}}^{j}\right\|_{L^{2}(\mathbb{P})}^{2}\right) .
$$

Indeed, using the fact that $u_{\lambda}^{j}$ solves the resolvent equation (2.2) we have

$$
\begin{aligned}
\left\|u_{\lambda}^{j}-u_{\lambda^{\prime}}^{j}\right\|_{\mathcal{H}_{1}}^{2} & =\left\langle u_{\lambda}^{j}-u_{\lambda^{\prime}}^{j},(-L)\left(u_{\lambda}^{j}-u_{\lambda^{\prime}}^{j}\right)\right\rangle_{L^{2}(\mathbb{P})}=\left\langle u_{\lambda}^{j}-u_{\lambda^{\prime}}^{j},-\left(\lambda u_{\lambda}^{j}-\lambda^{\prime} u_{\lambda^{\prime}}^{j}\right)\right\rangle_{L^{2}(\mathbb{P})} \\
& =-\lambda\left\|u_{\lambda}^{j}\right\|_{L^{2}(\mathbb{P})}^{2}-\lambda^{\prime}\left\|u_{\lambda^{\prime}}^{j}\right\|_{L^{2}(\mathbb{P})}^{2}+\left(\lambda+\lambda^{\prime}\right)\left\langle u_{\lambda}^{j}, u_{\lambda^{\prime}}^{j}\right\rangle_{L^{2}(\mathbb{P})} \\
& \leq 2 \sqrt{\lambda \lambda^{\prime}}\left\|u_{\lambda}^{j}\right\|_{L^{2}(\mathbb{P})}\left\|u_{\lambda^{\prime}}^{j}\right\|_{L^{2}(\mathbb{P})}+\left(\lambda+\lambda^{\prime}\right)\left\langle u_{\lambda}^{j}, u_{\lambda^{\prime}}^{j}\right\rangle_{L^{2}(\mathbb{P})} \\
& \leq\left(\sqrt{\lambda}+\sqrt{\lambda^{\prime}}\right)^{2}\left\|u_{\lambda}^{j}\right\|_{L^{2}(\mathbb{P})}\left\|u_{\lambda^{\prime}}^{j}\right\|_{L^{2}(\mathbb{P})},
\end{aligned}
$$

which gives (3.1). In particular, choosing $\lambda_{k}=2^{-k}$, we get

$$
\begin{aligned}
\left\|u_{\lambda_{k}}^{j}-u_{\lambda_{k-1}}^{j}\right\|_{\mathcal{H}_{1}}^{2} & \leq \frac{\left(\sqrt{2^{-k}}+\sqrt{2^{-k+1}}\right)^{2}}{2}\left(\left\|u_{\lambda_{k}}^{j}\right\|_{L^{2}(\mathbb{P})}^{2}+\left\|u_{\lambda_{k-1}}^{j}\right\|_{L^{2}(\mathbb{P})}^{2}\right) \\
& =\frac{(\sqrt{2}+1)^{2}}{2} \lambda_{k}\left(\left\|u_{\lambda_{k}}^{j}\right\|_{L^{2}(\mathbb{P})}^{2}+\left\|u_{\lambda_{k-1}}^{j}\right\|_{L^{2}(\mathbb{P})}^{2}\right) .
\end{aligned}
$$

Let now $k_{n}$ be the integer $k$ such that $2^{k-1} \leq n<2^{k}$. Then, we use the elementary estimate $\sqrt{a+b} \leq \sqrt{a}+\sqrt{b}$ for any $a, b \geq 0$ to obtain

$$
\left\|u_{\lambda_{k_{n}}}^{j}-u^{j}\right\|_{\mathcal{H}_{1}} \leq \sum_{m=k_{n}+1}^{\infty}\left\|u_{\lambda_{m}}^{j}-u_{\lambda_{m-1}}^{j}\right\|_{\mathcal{H}_{1}} \leq c \sum_{m=k_{n}+1}^{\infty} \sqrt{\lambda_{m}}\left(\left\|u_{\lambda_{m}}^{j}\right\|_{L^{2}(\mathbb{P})}+\left\|u_{\lambda_{m-1}}^{j}\right\|_{L^{2}(\mathbb{P})}\right) .
$$

Recall that $\left\|u_{\lambda_{m}}^{j}\right\|_{L^{2}(\mathbb{P})}=O\left(\lambda_{m}^{-\alpha}\right)$ by Proposition 3.2. Therefore, for $n$ large enough

$$
\left\|u_{\lambda_{k_{n}}}^{j}-u^{j}\right\|_{\mathcal{H}_{1}} \leq c \sum_{m=k_{n}+1}^{\infty} \lambda_{m}^{1 / 2-\alpha}=c \lambda_{k_{n}}^{1 / 2-\alpha}=c n^{\alpha-1 / 2} .
$$

Thus, the claim follows by choosing $\lambda_{k_{n}}$ for $\lambda$ in equation (2.13)-(2.15).

Recall that $\left(P_{t}\right)_{t \geq 0}$ denotes the transition semigroup of the environment process $\eta$.

Lemma 3.3. Under the assumptions of Theorem 1.3 or Theorem 1.4, there exists $\alpha<1 / 2$ such that for every $j=1, \ldots, d$,

$$
\left\|\int_{0}^{t} P_{s} V_{j} d s\right\|_{L^{2}(\mathbb{P})} \leq c(1 \vee t)^{\alpha}
$$

Lemma 3.3, which will be proven in the next subsection, immediately implies Proposition 3.2.

Proof of Proposition 3.2. Since $u_{\lambda}^{j}$ is the solution of the resolvent equation (2.2),

$$
u_{\lambda}^{j}=\int_{0}^{\infty} e^{-\lambda s} P_{s} V_{j} d s=\lambda \int_{0}^{\infty} \int_{s}^{\infty} e^{-\lambda t} P_{s} V_{j} d t d s=\lambda \int_{0}^{\infty} e^{-\lambda t} \int_{0}^{t} P_{s} V_{j} d s d t
$$


Hence, by Lemma 3.3 we get that

$$
\left\|u_{\lambda}^{j}\right\|_{L^{2}(\mathbb{P})} \leq c_{1} \lambda \int_{0}^{\infty} e^{-\lambda t}(1 \vee t)^{\alpha} d t \leq c_{1}+c_{1} \lambda \int_{1}^{\infty} e^{-\lambda t} t^{\alpha} d t \leq c_{1}+c_{1} \Gamma(\alpha+1) \lambda^{-\alpha},
$$

which is the claim.

\subsection{A Two-Walk Estimate}

In this subsection we prove Lemma 3.3. We shall use techniques from [JR, Section 3], [RS2, Appendix A] and [Mou]. Denote by $\left(X_{t}\right)_{t}$ and $\left(\tilde{X}_{t}\right)_{t}$ two independent random walks evolving in the same environment $\omega$ both starting from zero. We will write $\mathbb{P}_{2, x, \tilde{x}}$ in short for the averaged law of $(X, \tilde{X})$ starting in $(x, \tilde{x}) \in \mathbb{Z}^{d} \times \mathbb{Z}^{d}$ and $\mathbb{E}_{2, x, \tilde{x}}$ for the corresponding expectation, i.e. $\mathbb{E}_{2, x, \tilde{x}}=\mathbb{E} \otimes E_{0, x}^{\omega} \otimes E_{0, \tilde{x}}^{\omega}$. For abbreviation we will write $\mathbb{P}_{2, x}=\mathbb{P}_{2, x, 0}$ and $\mathbb{E}_{2, x}=\mathbb{E}_{2, x, 0}$ as well as $\mathbb{P}_{2}=\mathbb{P}_{2,0,0}$ and $\mathbb{E}_{2}=\mathbb{E}_{2,0,0}$. Furthermore, let $\left(Y_{t}\right)_{t \geq 0}$ be the continuous time Markov chain evolving in an environment $\omega$ with transition probabilities given by

$$
\pi_{s, x}^{\omega}\left[Y_{t} \in A\right]=P^{\omega}\left[Y_{t} \in A \mid Y_{s}=x\right]=\sum_{u, v \in \mathbb{Z}^{d}} \mathbb{1}_{\{v-u \in A\}} p^{\omega}(s, 0 ; t, u) p^{\omega}(s, x ; t, v) .
$$

The corresponding expectation will be denoted by $E_{s, x}^{\pi, \omega}$. In particular, note that for every $\omega$ the law of $X_{t}-\tilde{X}_{t}$ induced by $E_{x}^{\omega} \otimes E_{0}^{\omega}$ is the same as that of $Y_{t}$.

Lemma 3.4. For any $0 \leq s \leq t$ with $t-s \geq 1, y \in \mathbb{Z}^{d}$ and any ball $B(x, r)$ we have

$$
\pi_{s, y}^{\omega}\left[Y_{t} \in B(x, r)\right] \leq c(t-s)^{-d / 2} r^{d} .
$$

Proof. By the heat kernel estimates in Proposition 1.1 we have

$$
\begin{aligned}
\pi_{s, y}^{\omega}\left[Y_{t} \in B(x, r)\right] & =\sum_{u, v \in \mathbb{Z}^{d}} \mathbb{1}_{\{v-u \in B(x, r)\}} p^{\omega}(s, 0 ; t, u) p^{\omega}(s, y ; t, v) \\
& =\sum_{u \in \mathbb{Z}^{d}} p^{\omega}(s, 0 ; t, u) \sum_{v \in \mathbb{Z}^{d}} \mathbb{1}_{\{v \in B(x+u, r)\}} p^{\omega}(s, y ; t, v) \\
& \leq c(t-s)^{-d / 2} r^{d}
\end{aligned}
$$

which is the claim.

\subsubsection{Proof of Lemma 3.3 under Assumptions A4 and A5}

Let $d \geq 3$ and assume that A1-A5 hold. It is enough to show that there exists $\beta>1 / 2$ such that for every $j=1, \ldots, d$,

$$
\left\|P_{t} V_{j}\right\|_{L^{2}(\mathbb{P})} \leq c(1 \vee t)^{-\beta}
$$


First note that by definition $V_{j}(\omega)=\mu_{0, e_{j}}^{\omega}(0)-\mu_{0,-e_{j}}^{\omega}(0)$, so by Assumptions A1 and A3 we have $\mathbb{E}\left[V_{j}\right]=0$ and $\left\|V_{j}\right\|_{L^{\infty}(\mathbb{P})} \leq 2 C_{u}$, respectively. In particular, it remains to prove (3.2) for $t \geq 1$. Setting

$$
S_{n}(f):=\sum_{x \in B(0, n)} f\left(\tau_{0, x} \omega\right)
$$

we have by the translation invariance of $\mathbb{P}$

$$
\mathbb{E}\left[\left(S_{n}\left(P_{t} V_{j}\right)\right)^{2}\right]=\sum_{x, y \in B(0, n)} \mathbb{E}\left[P_{t} V_{j}\left(\tau_{0, x-y} \omega\right) P_{t} V_{j}(\omega)\right]=\sum_{x, y \in B(0, n)} \mathbb{E}_{2, x-y}\left[V_{j}\left(\tau_{t, X_{t}} \omega\right) V_{j}\left(\tau_{t, \tilde{X}_{t}} \omega\right)\right] .
$$

Let $\kappa>0$ to be chosen below. Then, for every $z \in \mathbb{Z}^{d}$ we use Lemma 3.4 and Assumption A5 and obtain

$$
\begin{aligned}
\mathbb{E}_{2, z}\left[V_{j}\left(\tau_{t, X_{t}} \omega\right) V_{j}\left(\tau_{t, \tilde{X}_{t}} \omega\right)\right] & \leq \mathbb{E}_{2, z}\left[V_{j}\left(\tau_{t, X_{t}} \omega\right) V_{j}\left(\tau_{t, \tilde{X}_{t}} \omega\right) \mathbb{1}_{\left\{\left|\tilde{X}_{t}-X_{t}\right|>n^{\kappa}\right\}}\right]+c \mathbb{P}_{2, z}\left[\left|Y_{t}\right| \leq n^{\kappa}\right] \\
& \leq \mathbb{E}_{2, z}\left[\mathbb{E}_{2, z}\left[V_{j}\left(\tau_{t, X_{t}} \omega\right) V_{j}\left(\tau_{t, \tilde{X}_{t}} \omega\right) \mid X_{t}, \tilde{X}_{t}\right] \mathbb{1}_{\left\{\left|\tilde{X}_{t}-X_{t}\right|>n^{\kappa}\right\}}\right]+c t^{-d / 2} n^{\kappa d} \\
& =\mathbb{E}_{2, z}\left[\mathbb{E}\left[V_{j}\left(\tau_{t, X_{t}} \omega\right) V_{j}\left(\tau_{t, \tilde{X}_{t}} \omega\right)\right] \mathbb{1}_{\left\{\left|\tilde{X}_{t}-X_{t}\right|>n^{\kappa}\right\}}\right]+c t^{-d / 2} n^{\kappa d} \\
& \leq c\left(n^{-\kappa p_{2}}+t^{-d / 2} n^{\kappa d}\right)
\end{aligned}
$$

Hence,

$$
n^{-2 d} \mathbb{E}\left[\left(S_{n}\left(P_{t} V_{j}\right)\right)^{2}\right] \leq c\left(n^{-\kappa p_{2}}+t^{-d / 2} n^{\kappa d}\right) .
$$

Next we rewrite the Dirichlet form of the process $\eta$ as

$$
\begin{aligned}
& \left\langle P_{t} V_{j}, P_{t} V_{j}\right\rangle_{\mathcal{H}_{1}}=\frac{1}{2} \sum_{y \in \mathbb{Z}^{d}} \mathbb{E}\left[\mu_{0 y}^{\omega}(0)\left(P_{t} V_{j}\left(\tau_{0, y} \omega\right)-P_{t} V_{j}(\omega)\right)^{2}\right] \\
= & \frac{1}{2} \sum_{y \in \mathbb{Z}^{d}} \mathbb{E}\left[\mu_{0 y}^{\omega}(0)\left(E_{0, y}^{\omega}\left[V_{j}\left(\tau_{t, X_{t}} \omega\right)\right]-E_{0,0}^{\omega}\left[V_{j}\left(\tau_{t, X_{t}} \omega\right)\right]\right)\left(E_{0, y}^{\omega}\left[V_{j}\left(\tau_{t, \tilde{X}_{t}} \omega\right)\right]-E_{0,0}^{\omega}\left[V_{j}\left(\tau_{t, \tilde{X}_{t}} \omega\right)\right]\right)\right] \\
= & \frac{1}{2} \sum_{y \in \mathbb{Z}^{d}}\left(\mathbb{E}_{2, y, y}\left[\mu_{0 y}^{\omega}(0) V_{j}\left(\tau_{t, X_{t}} \omega\right) V_{j}\left(\tau_{t, \tilde{X}_{t}} \omega\right)\right]-\mathbb{E}_{2, y, 0}\left[\mu_{0 y}^{\omega}(0) V_{j}\left(\tau_{t, X_{t}} \omega\right) V_{j}\left(\tau_{t, \tilde{X}_{t}} \omega\right)\right]\right. \\
& \left.-\mathbb{E}_{2,0, y}\left[\mu_{0 y}^{\omega}(0) V_{j}\left(\tau_{t, X_{t}} \omega\right) V_{j}\left(\tau_{t, \tilde{X}_{t}} \omega\right)\right]+\mathbb{E}_{2,0,0}\left[\mu_{0 y}^{\omega}(0) V_{j}\left(\tau_{t, X_{t}} \omega\right) V_{j}\left(\tau_{t, \tilde{X}_{t}} \omega\right)\right]\right) .
\end{aligned}
$$

Then, by the time mixing in Assumption A4 we have

$$
\begin{aligned}
\mathbb{E}_{2, y, y}\left[\mu_{0 y}^{\omega}(0) V_{j}\left(\tau_{t, X_{t}} \omega\right) V_{j}\left(\tau_{t, \tilde{X}_{t}} \omega\right)\right] & =\mathbb{E}_{2, y, y}\left[\mathbb{E}_{2, y, y}\left[\mu_{0 y}^{\omega}(0) V_{j}\left(\tau_{t, X_{t}} \omega\right) V_{j}\left(\tau_{t, \tilde{X}_{t}} \omega\right) \mid X_{t}, \tilde{X}_{t}\right]\right] \\
& =\mathbb{E}_{2, y, y}\left[\mathbb{E}\left[\mu_{0 y}^{\omega}(0) V_{j}\left(\tau_{t, X_{t}} \omega\right) V_{j}\left(\tau_{t, \tilde{X}_{t}} \omega\right)\right]\right] \\
& \leq \mathbb{E}_{2, y, y}\left[\mathbb{E}\left[\mu_{0 y}^{\omega}(0)\right] \mathbb{E}\left[V_{j}\left(\tau_{t, X_{t}} \omega\right) V_{j}\left(\tau_{t, \tilde{X}_{t}} \omega\right)\right]\right]+c t^{-p_{1}} \\
& =\mathbb{E}\left[\mu_{0 y}^{\omega}(0)\right] \cdot \mathbb{E}_{2,0,0}\left[\mathbb{E}\left[V_{j}\left(\tau_{t, X_{t}} \omega\right) V_{j}\left(\tau_{t, \tilde{X}_{t}} \omega\right)\right]\right]+c t^{-p_{1}} \\
& \leq c\left(n^{-\kappa p_{2}}+t^{-d / 2} n^{\kappa d}+t^{-p_{1}}\right),
\end{aligned}
$$

where we also used Assumption A1 in the fourth step and (3.3) in the last step. The other three terms in (3.5) can be treated similarly, and we obtain that

$$
\left\langle P_{t} V_{j}, P_{t} V_{j}\right\rangle_{\mathcal{H}_{1}} \leq c\left(n^{-\kappa p_{2}}+t^{-d / 2} n^{\kappa d}+t^{-p_{1}}\right) .
$$


Note that by the ellipticity in Assumption A3 for any $f \in \mathcal{D}(L)$ the Dirichlet form $\langle f, f\rangle_{\mathcal{H}_{1}}$ is comparable with the Dirichlet form of the environment process associated with a simple random walk on $\mathbb{Z}^{d}$. Thus, by Proposition 3.2 in [Mou], which is a simple consequence of the local Poincaré inequality on $\mathbb{Z}^{d}$, there exists $C_{S}>0$ such that for any $f \in \mathcal{D}(L)$ and $n \in \mathbb{N}$,

$$
\mathbb{E}\left[f(\omega)^{2}\right] \leq C_{S} n^{2}\langle f, f\rangle_{\mathcal{H}_{1}}+\frac{2}{|B(0, n)|^{2}} \mathbb{E}\left[S_{n}(f)^{2}\right] .
$$

Combining this with (3.4) and (3.6) we get

$$
\begin{aligned}
\mathbb{E}\left[P_{t} V_{j}(\omega)^{2}\right] & \leq C_{S} n^{2}\left\langle P_{t} V_{j}, P_{t} V_{j}\right\rangle_{\mathcal{H}_{1}}+\frac{2}{|B(0, n)|^{2}} \mathbb{E}\left[S_{n}\left(P_{t} V_{j}\right)^{2}\right] \\
& \leq c\left(n^{2-\kappa p_{2}}+t^{-d / 2} n^{2+\kappa d}+t^{-p_{1}} n^{2}\right)
\end{aligned}
$$

By Assumption A5 we have $p_{2}>2 d /(d-2)$, so there exists $\delta>0$ such that $p_{2}>$ $(1+\delta) 2 d /(d-2)$. Now let

$$
\kappa>\max \left(\frac{2+\frac{4(1+\delta)}{d-2}}{p_{2}-\frac{2(1+\delta) d}{d-2}}, \frac{1}{d}\left(\frac{d-2}{p_{1}-1}-2\right)\right)
$$

and

$$
\varrho \in\left(\frac{1}{1+\delta} \frac{d / 2-1}{\kappa d+2}, \frac{d / 2-1}{\kappa d+2}\right) .
$$

Finally, choosing $n=t^{\varrho}$ in (3.7) gives (3.2).

\subsubsection{Proof of Lemma 3.3 under Assumptions A4' and A5'}

Let $d \geq 1$ and assume that A1-A3, A4' and A5' hold. Notice first that

$$
\begin{aligned}
\left\|\int_{0}^{t} P_{s} V_{j} d s\right\|_{L^{2}(\mathbb{P})}^{2} & =\int_{0}^{t} \int_{0}^{t} \mathbb{E}\left[\sum_{x, y \in \mathbb{Z}^{d}} V_{j}\left(\tau_{r, x} \omega\right) V_{j}\left(\tau_{s, y} \omega\right) p^{\omega}(0,0 ; r, x) p^{\omega}(0,0 ; s, y)\right] d r d s \\
& =\int_{0}^{t} \int_{0}^{t} \mathbb{E}\left[E_{0,0}^{\omega}\left[V_{j}\left(\tau_{r, X_{r}} \omega\right)\right] E_{0,0}^{\omega}\left[V_{j}\left(\tau_{s, \tilde{X}_{s}} \omega\right)\right]\right] d r d s \\
& =2 \int_{0}^{t} \int_{0}^{t} \mathbb{1}_{\{r \leq s\}} \mathbb{E}\left[E_{0,0}^{\omega}\left[V_{j}\left(\tau_{r, X_{r}} \omega\right)\right] E_{0,0}^{\omega}\left[V_{j}\left(\tau_{s, \tilde{X}_{s}} \omega\right)\right]\right] d r d s
\end{aligned}
$$

and that by definition $V_{j}(\omega)=\mu_{0, e_{j}}^{\omega}(0)-\mu_{0,-e_{j}}^{\omega}(0)$, so by Assumption A1 and A3 we have $\mathbb{E}\left[V_{j}\right]=0$ and $\left\|V_{j}\right\|_{L^{\infty}(\mathbb{P})} \leq 2 C_{u}$, respectively. Again it suffices to consider $t \geq 1$.

For any $0 \leq r<s \leq t$ with $s-r \geq 1$ we have by Assumption A4'

$$
\begin{aligned}
\mathbb{E} E_{0,0}^{\omega}\left[V_{j}\left(\tau_{r, X_{r}} \omega\right)\right] E_{0,0}^{\omega}\left[V_{j}\left(\tau_{s, \tilde{X}_{s}} \omega\right)\right] & =\mathbb{E}_{2}\left[\mathbb{E}_{2}\left[V_{j}\left(\tau_{r, X_{r}} \omega\right) V_{j}\left(\tau_{s, \tilde{X}_{s}} \omega\right) \mid X_{r}, \tilde{X}_{s}\right]\right] \\
& =\mathbb{E}_{2} \mathbb{E}\left[V_{j}\left(\tau_{r, X_{r}} \omega\right) V_{j}\left(\tau_{s, \tilde{X}_{s}} \omega\right)\right] \\
& \leq c(s-r)^{-p_{1}}
\end{aligned}
$$


with $p_{1}>d+1$ if $d \geq 2$ and $p_{1}>4$ if $d=1$. We fix $\delta \in\left(1 / p_{1}, 1 /(d+1)\right)$ if $d \geq 2$ and $\delta \in\left(1 / p_{1}, 1 / 4\right)$ if $d=1$ and set $T_{t}:=t^{\delta}$ and $L_{t}:=\left(1 \vee c_{1}\right) T_{t}$ (with constant $c_{1}$ as in Proposition 1.1). Then,

$$
\int_{0}^{t} \int_{0}^{t} \mathbb{1}_{\{r \leq s\}} \mathbb{1}_{\left\{s-r \geq T_{t}\right\}} \mathbb{E}\left[E_{0,0}^{\omega}\left[V_{j}\left(\tau_{r, X_{r}} \omega\right)\right] E_{0,0}^{\omega}\left[V_{j}\left(\tau_{s, \tilde{X}_{s}} \omega\right)\right]\right] d r d s \leq c t^{2} T_{t}^{-p_{1}} .
$$

Now we shall consider pairs of times $r$ and $s$ with distance less than $T_{t}$. We decompose the integral as follows.

$$
\begin{aligned}
& \int_{0}^{t} \int_{0}^{t} \mathbb{1}_{\{r \leq s\}} \mathbb{1}_{\left\{s-r<T_{t}\right\}} \mathbb{E}\left[E_{0,0}^{\omega}\left[V_{j}\left(\tau_{r, X_{r}} \omega\right)\right] E_{0,0}^{\omega}\left[V_{j}\left(\tau_{s, \tilde{X}_{s}} \omega\right)\right]\right] d r d s \\
\leq & \int_{0}^{t} \int_{0}^{t} \mathbb{1}_{\{r \leq s\}} \mathbb{1}_{\left\{s-r<T_{t}\right\}} \mathbb{E}_{2}\left[V_{j}\left(\tau_{r, X_{r}} \omega\right) V_{j}\left(\tau_{s, \tilde{X}_{s}} \omega\right) \mathbb{1}_{\left\{\left|X_{r}-\tilde{X}_{r}\right|>2 L_{t}\right\}}\right] d r d s \\
& +c T_{t} \int_{0}^{t} \mathbb{P}_{2}\left[\left|X_{r}-\tilde{X}_{r}\right| \leq 2 L_{t}\right] d r \\
\leq & \int_{0}^{t} \int_{0}^{t} \mathbb{1}_{\{r \leq s\}} \mathbb{1}_{\left\{s-r<T_{t}\right\}} \mathbb{E}_{2}\left[V_{j}\left(\tau_{r, X_{r}} \omega\right) V_{j}\left(\tau_{s, \tilde{X}_{s}} \omega\right) \mathbb{1}_{\left\{\left|X_{r}-\tilde{X}_{s}\right|>L_{t}\right\}} \mathbb{1}_{\left\{\left|X_{r}-\tilde{X}_{r}\right|>2 L_{t}\right\}}\right] d r d s \\
& +c \int_{0}^{t} \int_{0}^{t} \mathbb{1}_{\{r \leq s\}} \mathbb{1}_{\left\{s-r<T_{t}\right\}} \mathbb{P}_{2}\left[\left|X_{r}-\tilde{X}_{s}\right| \leq L_{t},\left|X_{r}-\tilde{X}_{r}\right|>2 L_{t}\right] d r d s \\
& +c T_{t} \int_{0}^{t} \mathbb{E} \pi_{0,0}^{\omega}\left[\left|Y_{r}\right| \leq 2 L_{t}\right] d r .
\end{aligned}
$$

To estimate the first term in (3.9) note that conditioned on the event $\left\{\left|X_{r}-\tilde{X}_{s}\right|>L_{t}\right\}$ we have that $V_{j}\left(\tau_{r, X_{r}} \omega\right)$ and $V_{j}\left(\tau_{s, \tilde{X}_{s}} \omega\right)$ depend only on variables contained in two subsets of $\mathbb{Z}^{d}$ with distance $L_{t}$. Thus, by Assumption A5' we obtain

$$
\begin{aligned}
& \mathbb{E}_{2}\left[V_{j}\left(\tau_{r, X_{r}} \omega\right) V_{j}\left(\tau_{s, \tilde{X}_{s}} \omega\right) \mathbb{1}_{\left\{\left|X_{r}-\tilde{X}_{s}\right|>L_{t}\right\}} \mathbb{1}_{\left\{\left|X_{r}-\tilde{X}_{r}\right|>2 L_{t}\right\}}\right] \\
= & \mathbb{E}_{2}\left[\mathbb{E}_{2}\left[V_{j}\left(\tau_{r, X_{r}} \omega\right) V_{j}\left(\tau_{s, \tilde{X}_{s}} \omega\right) \mid X_{r}, \tilde{X}_{r}, \tilde{X}_{s}\right] \mathbb{1}_{\left\{\left|X_{r}-\tilde{X}_{s}\right|>L_{t}\right\}} \mathbb{1}_{\left\{\left|X_{r}-\tilde{X}_{r}\right|>2 L_{t}\right\}}\right] \\
= & \mathbb{E}_{2}\left[\mathbb{E}\left[V_{j}\left(\tau_{r, X_{r}} \omega\right) V_{j}\left(\tau_{s, \tilde{X}_{s}} \omega\right)\right] \mathbb{1}_{\left\{\left|X_{r}-\tilde{X}_{s}\right|>L_{t}\right\}} \mathbb{1}_{\left\{\left|X_{r}-\tilde{X}_{r}\right|>2 L_{t}\right\}}\right] \\
\leq & c L_{t}^{-p_{2}}
\end{aligned}
$$

Next we estimate the second term in (3.9). First we use the Markov property to get

$$
\mathbb{P}_{2}\left[\left|X_{r}-\tilde{X}_{s}\right| \leq L_{t},\left|Y_{r}\right|>2 L_{t}\right] \leq \mathbb{P}_{2}\left[\left|\tilde{X}_{s}-\tilde{X}_{r}\right|>L_{t}\right]=\mathbb{P} P_{0,0}^{\omega} P_{r, \tilde{X}_{r}}^{\omega}\left[\left|\tilde{X}_{s}-\tilde{X}_{r}\right|>L_{t}\right]
$$

Set $D_{i}:=\left\{y \in \mathbb{Z}^{d}: 2^{i} L_{t} \leq\left|y-\tilde{X}_{r}\right| \leq 2^{i+1} L_{t}\right\}, i \geq 0$. Then, noting that $s-r \leq T_{t} \leq$ 
$c_{1}^{-1} L_{t}$, we use the heat kernel estimates in Proposition 1.1 to obtain

$$
\begin{aligned}
P_{r, \tilde{X}_{r}}^{\omega}\left[\left|\tilde{X}_{s}-\tilde{X}_{r}\right|>L_{t}\right] & =\sum_{y \in B\left(\tilde{X}_{r}, L_{t}\right)^{c}} p^{\omega}\left(r, \tilde{X}_{r} ; s, y\right)=\sum_{i=0}^{\infty} \sum_{y \in D_{i}} p^{\omega}\left(r, \tilde{X}_{r} ; s, y\right) \\
& \leq c \sum_{i=0}^{\infty} \sum_{y \in D_{i}} \exp \left(-c\left|y-\tilde{X}_{r}\right| \log \left(\left|y-\tilde{X}_{e}\right| /(s-r)\right)\right) \\
& \leq c \sum_{i=0}^{\infty}\left(2^{i} L_{t}\right)^{d} \exp \left(-c 2^{i} L_{t} \log \left(2^{i} L_{t} / T_{t}\right)\right) \\
& \leq c \sum_{i=0}^{\infty}\left(2^{i} L_{t}\right)^{d} \exp \left(-c 2^{i} L_{t}\right) .
\end{aligned}
$$

An elementary computation now gives

$$
\mathbb{P}_{2}\left[\left|X_{r}-\tilde{X}_{s}\right|>L_{t},\left|Y_{r}\right| \leq 2 L_{t}\right] \leq c L_{t}^{d} \int_{1}^{\infty} \exp \left(-c L_{t} u\right) d u \leq c L_{t}^{d-1} \exp \left(-c L_{t}\right) .
$$

To estimate the last term in (3.9) we use Lemma 3.4 to obtain in the case $d \geq 2$

$$
\int_{0}^{t} \pi_{0,0}^{\omega}\left[\left|Y_{r}\right| \leq 2 L_{t}\right] d r \leq \int_{0}^{t}(1 \vee r)^{-d / 2} L_{t}^{d} d r \leq c L_{t}^{d} \int_{0}^{t}(1 \vee r)^{-1} d r \leq c \log t L_{t}^{d}
$$

and if $d=1$

$$
\int_{0}^{t} \pi_{0,0}^{\omega}\left[\left|Y_{r}\right| \leq 2 L_{t}\right] d r \leq \int_{0}^{t}(1 \vee r)^{-1 / 2} L_{t}^{d} d r \leq c t^{1 / 2} L_{t}
$$

Finally, combining (3.8) and (3.9) we get in the case $d \geq 2$ by (3.10), (3.11) and (3.12)

$$
\left\|\int_{0}^{t} P_{s} V_{j}\right\|_{L^{2}(\mathbb{P})}^{2} d s \leq c\left(t^{2} T_{t}^{-p_{1}}+t T_{t} L_{t}^{-p_{2}}+t T_{t} L_{t}^{d-1} \exp \left(-c L_{t}\right)+T_{t} \log t L_{t}^{d}\right) .
$$

Analogously, if $d=1$ we obtain by (3.10), (3.11) and (3.13) that

$$
\left\|\int_{0}^{t} P_{s} V_{j}\right\|_{L^{2}(\mathbb{P})}^{2} d s \leq c\left(t^{2} T_{t}^{-p_{1}}+t T_{t} L_{t}^{-p_{2}}+t T_{t} \exp \left(-c L_{t}\right)+t^{1 / 2} T_{t} L_{t}\right) .
$$

The claim follows by our choice of $\delta, L_{t}$ and $T_{t}$.

\section{Invariance Principle for $X$}

In this section we prove the annealed FCLT in Theorem 1.2 and the quenched FCLT in Theorem 1.3 and Theorem 1.4, respectively. Throughout this section we suppose that Assumptions A1-A3 hold. The first step to prove a quenched invariance principle for the random walk $X$ is to show that the processes $X^{(\varepsilon)}$ are tight. 
Theorem 4.1. Let $T>0, r>0$. Then

$$
\begin{aligned}
& \lim _{R \rightarrow \infty} \sup _{\varepsilon} P_{0,0}^{\omega}\left(\sup _{s \leq T}\left|X_{s}^{(\varepsilon)}\right|>R\right) \rightarrow 0, \\
& \lim _{\delta \rightarrow 0} \limsup _{\varepsilon \rightarrow 0} P_{0,0}^{\omega}\left(\sup _{\left|s_{1}-s_{2}\right| \leq \delta, s_{i} \leq T}\left|X_{s_{2}}^{(\varepsilon)}-X_{s_{1}}^{(\varepsilon)}\right|>r\right)=0 .
\end{aligned}
$$

Proof. From the heat kernel estimates in Proposition 1.1 one can derive tail estimates for the exit times of $X$ from balls (see e.g. [ABDH, Proposition 4.7]). Then tightness follows by the same arguments as in $\mathrm{ABDH}$, Proposition 5.13].

For $n \in \mathbb{N}$ let $\widehat{X}_{n}=X_{n}$, and set

$$
\widehat{X}_{t}^{(\varepsilon)}=\varepsilon \widehat{X}_{\left\lfloor t / \varepsilon^{2}\right\rfloor}, \quad 0<\varepsilon \leq 1 .
$$

Lemma 4.2. For any $u>0$,

$$
\lim _{\varepsilon \rightarrow 0} P_{0,0}^{\omega}\left(\sup _{0 \leq s \leq T}\left|\widehat{X}_{s}^{(\varepsilon)}-X_{s}^{(\varepsilon)}\right|>u\right)=0
$$

Proof. This follows from the proof of Theorem 4.1 by the same arguments as in [BD, Lemma 4.12].

We will first establish the convergence of the processes $\widehat{X}^{(\varepsilon)}$; using Lemma 4.2 will then give the convergence of $X^{(\varepsilon)}$ to the same limit. We define

$$
\widehat{M}_{n}=M_{n}, \quad \widehat{M}_{t}^{(\varepsilon)}=\varepsilon \widehat{M}_{\left\lfloor t / \varepsilon^{2}\right\rfloor}, \quad t \geq 0,
$$

so that

$$
\widehat{X}_{t}^{(\varepsilon)}=\varepsilon \widehat{X}_{\left\lfloor t / \varepsilon^{2}\right\rfloor}=\widehat{M}_{t}^{(\varepsilon)}+\varepsilon \chi\left(\left\lfloor t / \varepsilon^{2}\right\rfloor, \varepsilon^{-1} \widehat{X}_{t}^{(\varepsilon)}, \omega\right) .
$$

Thus it is sufficient to prove that the martingale $\widehat{M}^{(\varepsilon)}$ converges to a Brownian motion with a certain covariance matrix, and that the second term in (4.6) converges to zero in $P_{0,0}^{\omega}$-probability for $\mathbb{P}_{\text {-a.a. }} \omega$ (resp. in $\mathbb{P}_{0,0}^{*}$-probability) to get the quenched FCLT (resp. the annealed FCLT). For any $G: \mathbb{Z}^{d} \times \Omega \rightarrow \mathbb{R}$ we define

$$
\overline{\mathbb{E}}[G]=\sum_{y \sim 0} \mathbb{E}\left[\mu_{0 y}^{\omega}(0) G(y, \omega)\right]
$$

Proposition 4.3. For $\mathbb{P}$-a.e. $\omega$, the sequence of processes $\left(\widehat{M}^{(\varepsilon)}\right)$ converges in law in the Skorohod topology to a Brownian motion with a non-degenerate covariance matrix $\Sigma$ given by $\Sigma_{i j}=\overline{\mathbb{E}} \Phi_{i} \Phi_{j}$.

Proof. We proceed as in $[\mathrm{BB}]$. Let $v \in \mathbb{R}^{d}$ be a unit vector, write as before $\widehat{M}_{n}^{v}=v \cdot M_{n}$, and let

$$
F_{K}(\omega)=E_{\omega}^{0}\left(\left|\widehat{M}_{1}^{v}\right|^{2} ;\left|\widehat{M}_{1}^{v}\right| \geq K\right)
$$


Then $F_{K}$ is decreasing in $K$, in particular $\mathbb{E}\left[F_{K}\right] \leq \mathbb{E}\left[F_{0}\right]$. In the notation of Corollary 2.10 $F_{0}(\omega)=\|v \cdot \Phi\|_{\omega}^{2}$, and so by (2.11) the covariance process of $\widehat{M}^{v}$ is

$$
\left\langle\widehat{M}^{v}\right\rangle_{n}=\int_{0}^{n} F_{0}\left(\eta_{s}\right) d s .
$$

So by the ergodicity of the environment process $\eta$ w.r.t. $\mathbb{P}$ we have $n^{-1}\left\langle\widehat{M}^{v}\right\rangle_{n} \rightarrow \mathbb{E}\left[F_{0}\right]$ as $n \rightarrow \infty, P_{0,0}^{\omega}$ a.s., for $\mathbb{P}$-a.a. $\omega$.

Using the same arguments as in [BB, Theorem 6.2] it is straightforward to check the conditions of the Lindeberg-Feller FCLT for martingales (see for example [Du, Theorem 3.4.5]), and deduce that $v \cdot \widehat{M}^{(\varepsilon)}$ converges to a real-valued Brownian motion with nonrandom covariance $\mathbb{E}\left[\|v \cdot \Phi\|_{\omega}^{2}\right]$, which can be written as $v \cdot \Sigma v$, where $\Sigma$ is the matrix with coefficients given by $\Sigma_{i j}=\overline{\mathbb{E}}\left[\Phi_{i} \Phi_{j}\right]$. By the Cramer-Wold Theorem (see e.g. Theorem 3.9 .5 in $[\mathrm{Du}]$ ) we get that $\widehat{M}^{(\varepsilon)}$ converges in law to an $\mathbb{R}^{d}$-valued Brownian motion with covariance matrix $\Sigma$.

It remains to show that $\Sigma$ is non-degenerate. By the uniform lower bound on the conductances in Assumption A3 we have for every unit vector $v \in \mathbb{R}^{d}$ that $v \cdot \Sigma v \geq v \cdot \Sigma_{C_{l}} v$, where $\Sigma_{C_{l}}$ denotes the non-degenerate covariance matrix of the limiting Brownian motion in the invariance principle for the simple random walk on $\mathbb{Z}^{d}$ with constant jump rate $C_{l}$. Thus, $v \cdot \Sigma v>0$, which implies that $\Sigma$ is positive-definite.

To conclude the proof of the invariance principles we need to control the corrector function. First we complete the proof of the annealed FCLT.

Proof of Theorem 1.2. Setting $R_{n}:=\chi\left(n, X_{n}, \omega\right)$ we need to show that

$$
n^{-1 / 2} \max _{k \leq n}\left|R_{k}\right| \rightarrow 0 \quad \text { in } \mathbb{P}_{0,0}^{*} \text {-probability as } n \rightarrow \infty
$$

By Proposition 2.11 we have that $n^{-1 / 2} R_{n}$ converges to 0 in $L^{2}\left(\mathbb{P}_{0,0}^{*}\right)$ and thus in $\mathbb{P}_{0,0^{*}}$ probability. By an elementary property of real convergent sequences, we get (4.7).

Finally, to complete the proof of the quenched invariance principle we prove

Proposition 4.4. Let $T>0$. Under the assumptions of Theorem 1.3 or Theorem 1.4, for $\mathbb{P}$-a.e. $\omega$,

$$
\sup _{t \leq T} \varepsilon \chi\left(\left\lfloor t / \varepsilon^{2}\right\rfloor, \varepsilon^{-1} \widehat{X}_{t}^{(\varepsilon)}, \omega\right) \rightarrow 0 \quad \text { in } P_{0,0}^{\omega}-\text { probability } .
$$

Proof. We will proceed as in [RS1] applying the theory of "fractional coboundaries" of Derriennic and Lin [DeLi]. Setting $R_{n}:=\chi\left(n, X_{n}, \omega\right)$ we need to show that

$$
n^{-1 / 2} \max _{k \leq n}\left|R_{k}\right| \rightarrow 0 \quad \text { in } P_{0,0}^{\omega} \text {-probability as } n \rightarrow \infty \text {. }
$$


Let $\tilde{\mathbb{P}}$ denote the path measure on $\Omega^{\mathbb{N}}$ of the random sequence $\left(\tau_{n, X_{n}} \omega\right)_{n \geq 0}$ with initial distribution $\mathbb{P}$, and let $\theta$ be the shift map on the sequence space $\Omega^{\mathbb{N}}$. By the cocycle property in Corollary 2.9 we have $\chi(0,0, \omega)=0$ and hence

$$
R_{n}=\sum_{k=0}^{n-1} \chi\left(k+1, X_{k+1}, \omega\right)-\chi\left(k, X_{k}, \omega\right)=\sum_{k=0}^{n-1} h\left(\tau_{k, X_{k}} \omega, \tau_{k+1, X_{k+1}} \omega\right)
$$

with $h$ defined as in Remark 2.8. For sequences $\bar{\omega}=\left(\omega^{(i)}\right)_{i \in \mathbb{N}}$ define $H(\bar{\omega})=h\left(\omega^{(0)}, \omega^{(1)}\right)$ and

$$
\tilde{R}_{n}=\sum_{k=0}^{n-1} H \circ \theta^{k}
$$

Then $H \in L^{2}(\tilde{\mathbb{P}})$ and the process $\left(\tilde{R}_{n}\right)$ has the same distribution under $\tilde{\mathbb{P}}$ as the process $\left(R_{n}\right)$ under $\mathbb{P} \otimes P_{0,0}^{\omega}$.

By Proposition 3.1 the assumptions of Theorem 2.17 in [DeLi] are satisfied. We conclude that $H \in(I-\theta)^{\gamma} L^{2}(\tilde{\mathbb{P}})$ for any $\gamma \in(0,1-\alpha)$. Since $\alpha<1 / 2$ there exists such a $\gamma \in(1 / 2,1-\alpha)$. Then, (i) in Theorem 3.2 in [DeLi] implies that $n^{-1 / 2} \tilde{R}_{n}$ converges to 0 , $\tilde{\mathbb{P}}$-a.s. Hence, $n^{-1 / 2} R_{n}$ converges to $0, \mathbb{P} \otimes P_{0,0}^{\omega}$-a.s. In other words, $n^{-1 / 2} R_{n}$ converges to $0, P_{0,0}^{\omega}$-a.s., for $\mathbb{P}$-a.e. $\omega$, which implies (4.8).

\section{Application to Stochastic Interface Models}

In this section we point out a relation between our results and the stochastic dynamic of an interface describing the separation of two pure thermodynamical phases, known as the Ginzburg Landau $\nabla \varphi$ model. We refer to [F] for a survey on these models. The interface is described by a field of height variables $\varphi_{t}(x), x \in \mathbb{Z}^{d}, t \geq 0$, whose stochastic dynamics are given by the following infinite system of stochastic differential equations involving nearest neighbour interaction:

$$
\varphi_{t}(x)=\varphi_{x}-\int_{0}^{t} \sum_{y:|x-y|=1} V^{\prime}\left(\varphi_{t}(x)-\varphi_{t}(y)\right) d t+\sqrt{2} w_{t}(x), \quad x \in \mathbb{Z}^{d} .
$$

Here $\varphi$ is the height of the interface at time $t=0,\left\{w(x), x \in \mathbb{Z}^{d}\right\}$ is a collection of independent Brownian motions and the potential $V \in C^{2}\left(\mathbb{R}, \mathbb{R}_{+}\right)$is even and strictly convex, i.e.

$$
c_{-} \leq V^{\prime \prime} \leq c_{+}
$$

for some $0<c_{-}<c_{+}<\infty$. Let for each $r>0$

$$
\mathcal{E}_{r}:=\left\{\varphi \in \mathbb{R}^{\mathbb{Z}^{d}}: \sum_{x}\left|\varphi_{x}\right|^{2} e^{-2 r|x|}<\infty\right\}
$$


denote the set of tempered configurations. Then, for every initial value $\varphi \in \mathcal{E}_{r}$ the SDE (5.1) admits a unique strong solution $\varphi_{t} \in \mathcal{E}_{r}, t \geq 0$, see [FS]. Let $H$ be the formal Hamiltonian given by

$$
H(\varphi)=\frac{1}{2} \sum_{y:|x-y|=1} V\left(\varphi_{x}-\varphi_{y}\right)
$$

then the formal equilibrium measure for the dynamic is given by the Gibbs measure

$$
\frac{1}{Z} \exp (-H(\varphi)) \prod_{x} d \varphi_{x}
$$

This can be made rigorous for the corresponding dynamic on a finite box. In dimension $d \geq 3$ Gibbs measures for the $\varphi$-field on the whole lattice can be constructed by taking the thermodynamical limit, cf. Section 4.5 in $[F]$. More precisely, for every $h \in \mathbb{R}$ there exists a shift-invariant and ergodic $\varphi$-Gibbs measure $m_{h}$ with mean $h$, i.e.

$$
\int \varphi_{x} m_{h}(d \varphi)=h, \quad x \in \mathbb{Z}^{d}
$$

These measures are also reversible and ergodic for the SDE (5.1). We denote by $\mathbb{P}_{m_{h}}$ the law of the process $\varphi_{t}$ started under the equilibrium distribution $m_{h}$ (and by $\mathbb{E}_{m_{h}}$ the corresponding expectation).

Next we consider discrete gradients, i.e. height differences of the form $\nabla_{b} \varphi=\varphi_{y_{b}}-\varphi_{x_{b}}$ for any bond $b=\left\{x_{b}, y_{b}\right\} \in E_{d}$. Then, as a vector field $\nabla \varphi$ has zero curl in the sense that

$$
\sum_{b \in \mathcal{C}} \nabla_{b} \varphi=0
$$

for every closed loop $\mathcal{C}$, i.e. the bonds $\left\{x_{i}, x_{i+1}\right\}$ of a sequence of $x_{0}, \ldots, x_{n}$ in $\mathbb{Z}^{d}$ satisfying $x_{0}=x_{n}$ and $\left|x_{i}-x_{i-1}\right|=1$ for $i \in\{1, \ldots n\}$. Let $\mathcal{X}$ be the subset of $\mathbb{R}^{E_{d}}$, whose elements have zero curl, and let for $r>0$

$$
\mathcal{X}_{r}=\left\{\eta \in \mathbb{R}^{E_{d}}: \eta_{b}=\nabla_{b} \varphi \text { for some } \varphi \in \mathcal{E}_{r}\right\}
$$

be the subset of tempered gradients. Note that the drift term in the SDE (5.1) can be rewritten as

$$
-\sum_{y:|x-y|=1} V^{\prime}(\varphi(x)-\varphi(y))=\sum_{b: x_{b}=x} V^{\prime}\left(\nabla_{b} \varphi\right)
$$

Then, for each initial $\nabla \varphi \in \mathcal{X}_{r}$, the gradient process $\left(\nabla_{b} \varphi_{t}, b \in E_{d}, t \geq 0\right)$ is the unique strong solution of the SDE

$$
\nabla_{b} \varphi_{t}=\nabla_{b} \varphi-\int_{0}^{t}\left(\sum_{b^{\prime}: x_{b^{\prime}}=x_{b}} V^{\prime}\left(\nabla_{b^{\prime}} \varphi_{s}\right)-\sum_{b^{\prime}: x_{b^{\prime}}=y_{b}} V^{\prime}\left(\nabla_{b^{\prime}} \varphi_{s}\right)\right) d s+\sqrt{2} \nabla_{b} w_{t}, \quad b \in E_{d}
$$

where $\nabla_{b} w_{t}=w_{t}\left(y_{b}\right)-w_{t}\left(x_{b}\right)$, see again [FS]. Also it has been shown in [FS, Theorems 3.1 and 3.2] that in any lattice dimension $d \geq 1$, given any $u \in \mathbb{R}^{d}$, there exists a unique shift invariant ergodic $\nabla \varphi$-Gibbs measure $\tilde{m}_{u}$ on $\mathcal{X}_{r}$ satisfying

$$
\int_{\mathcal{X}} \eta_{0, e_{i}} d \tilde{m}_{u}=u_{i}
$$


for every $i=1, \ldots, d$. Here $u$ is the tilt and $\tilde{m}_{u}$ the $u$-tilted measure. Moreover, $\tilde{m}_{u}$ is known to be an invariant reversible and ergodic measure for the gradient process $\nabla \varphi_{t}$ ([FS, Proposition 3.1]).

Our aim is to investigate the decay of the space-time correlation functions under the equilibrium Gibbs measures. The idea - originally from Helffer and Sjöstrand [HS] - is to describe the correlation functions in terms of a certain random walk in dynamic random environment (cf. also [DD, GOS, BG]). Let $\left(X_{t}\right)_{t \geq 0}$ be the random walk on $\mathbb{Z}^{d}$ with jump rates given by the random dynamic conductances

$$
\mu_{b}^{\nabla \varphi}(t):=V^{\prime \prime}\left(\nabla_{b} \varphi(t)\right)=V^{\prime \prime}\left(\varphi_{y_{b}}(t)-\varphi_{x_{b}}(t)\right), \quad b=\left\{x_{b}, y_{b}\right\} \in E_{d} .
$$

Since $V$ is even, the jump rates are symmetric, i.e. $\mu_{x_{b}, y_{b}}^{\nabla \varphi}(t)=\mu_{y_{b}, x_{b}}^{\nabla \varphi}(t)$. Further, let $p^{\nabla \varphi}(s, x ; t, y), x, y \in \mathbb{Z}^{d}, s \leq t$, denote the transition densities of the random walk $X$.

Theorem 5.1. $\quad$ i) Let $d \geq 3$ and let $m_{h}$ be any ergodic $\varphi$-Gibbs measure. Then, the environment $\mu^{\nabla \varphi}$ started under $m_{h}$ satisfies Assumptions A1-A3. Moreover, $\mu^{\nabla \varphi}$ also satisfies Assumptions A4 and A5 if $d \geq 6$.

ii) Let $d \geq 1$ and let $\tilde{m}_{u}$ be any ergodic $\nabla \varphi$-Gibbs measure. Then, the environment $\mu^{\nabla \varphi}$ started under $\tilde{m}_{u}$ satisfies Assumptions A1-A3. Moreover, $\mu^{\nabla \varphi}$ also satisfies Assumptions $A 4$ and $A 5$ if $d \geq 5$.

Proof. Assumption A1 is immediate from the ergodicity of the Gibbs measures $m_{h}$ and $\tilde{m}_{u}$, respectively. Assumption A2 is clear from the pathwise continuity of $\varphi_{t}$ and $\nabla \varphi_{t}$ and the strict convexity of $V$ in (5.2) guarantees the ellipticity in Assumption A3.

By Theorem 6.1 in [DD] the time-covariance under the $\varphi$-Gibbs measure $m_{h}$ decays polynomially with order $d / 2-1$ and the space-covariance decays polynomially with order $d-2$. Hence, Assumptions A4 and A5 hold if $d / 2-1>1$ and $d-2>2 d /(d-2)$ which is the case for $d \geq 6$.

On the other hand, by Theorem 6.2 in [DD] the time-covariance for $\nabla \varphi$ has polynomial decay of order $d / 2$ and the space-covariance has polynomial decay of order $d$. We have $d / 2>1$ and $d>2 d /(d-2)$ if $d \geq 5$.

We combine now the Helffer-Sjöstrand representation and the local limit theorem in Theorem 1.6 to get a scaling limit for the space-time covariation of the $\varphi$-field.

Theorem 5.2. Let $d \geq 3$ and let $m_{h}$ be any ergodic $\varphi$-Gibbs measure. Then, there exist a non-degenerate covariance matrix $\Sigma$ such that

$$
N^{d+2} \operatorname{cov}_{m_{h}}\left(\varphi_{0}(0), \varphi_{N^{2} t}(\lfloor N y\rfloor)\right) \rightarrow \int_{0}^{\infty} k_{t+s}^{(\Sigma)}(y) d s, \quad \text { as } N \rightarrow \infty
$$

where $k_{t}$ is the Gaussian kernel with diffusion matrix $\Sigma$ in (1.6).

Proof. By the Helffer-Sjöstrand representation (cf. equation (6.10) in [DD]) we have

$$
\operatorname{cov}_{m_{h}}\left(\varphi_{0}(0), \varphi_{t}(y)\right)=\int_{0}^{\infty} \mathbb{E}_{m_{h}}\left[p^{\nabla \varphi}(0,0 ; t+s, y)\right] d s
$$


Using the annealed local limit theorem in Theorem 1.6 i) we obtain

$$
\begin{aligned}
N^{d+2} \operatorname{cov}_{m_{h}}\left(\varphi_{0}(0), \varphi_{N^{2} t}(\lfloor N y\rfloor)\right) & =N^{d} \int_{0}^{\infty} \mathbb{E}_{m_{h}}\left[p^{\nabla \varphi}\left(0,0 ; N^{2}(t+s),\lfloor N y\rfloor\right)\right] d s \\
& \rightarrow \int_{0}^{\infty} k_{t+s}^{(\Sigma)}(y) d s
\end{aligned}
$$

as $N \rightarrow \infty$, which is the claim.

Ultimately, we would like to derive an analogous scaling limit for the space-time covariance of the gradient process $\nabla \varphi_{t}$, see also the discussion in [BG, Section 6]. However,

what is still missing until now is a local limit theorem for the gradient of the heat kernel.

\section{References}

[ABDH] S. Andres, M.T. Barlow, J.-D. Deuschel and B. Hambly. Invariance principle for the random conductance model, Preprint to appear in Probab. Theory Rel. Fields, available online DOI 10.1007/s00440-012-0435-2.

[BZ] A. Bandyopadhyay and O. Zeitouni. Random walk in dynamic Markovian random environment. ALEA, Lat. Am. J. Probab. Math. Stat. 1 (2006), 205-224.

[BD] M.T. Barlow and J.-D. Deuschel. Invariance principle for the random conductance model with unbounded conductances. Ann. Probab. 38 (2010), 234-276

[BH] M.T. Barlow and B.M. Hambly. Parabolic Harnack inequality and local limit theorem for percolation clusters. Elec. J. Prob. 14 (2009), Paper 1, 1-16.

[BB] N. Berger and M. Biskup. Quenched invariance principle for simple random walk on percolation clusters. Probab. Theory Rel. Fields 137 (2007), no. 1-2, 83-120.

[Bi] M. Biskup. Recent progress on the random concuctance model. Probab. Surv. 8 (2011), 294-373.

[BP] M. Biskup and T.M. Prescott. Functional CLT for random walk among bounded random conductances. Elec. J. Prob. 12 (2007), paper 49, 1323-1348.

[BG] T. Bodineau and B. Graham. Helffer-Sjöstrand representation for conservative dynamics. Markov Proc. Rel. Fields 18 (2012), 71-88.

[BMP1] C. Boldrighini, R. A. Minlos and A. Pellegrinotti. Random walks in quenched i.i.d. space-time random environment are always a.s. diffusive. Probab. Theory Related Fields 129 (2004), no. 1, 133-156.

[BMP2] C. Boldrighini, R. A. Minlos and A. Pellegrinotti. Discrete-time random motion in a continuous random medium. Stochastic Process. Appl. 119 (2009), no. 10, 32853299. 
[DD] T. Delmotte and J.-D. Deuschel. On estimating the derivatives of symmetric diffusions in stationary random environment, with applications to $\nabla \varphi$ interface model. Probab. Theory Related Fields 133 (2005), no. 3, 358-390.

[DeLi] Y. Derriennic and M. Lin. Fractional Poisson equations and ergodic theorems for fractional coboundaries. Israel J. Math. 123 (2001), 93-130.

[DoLi] D. Dolgopyat and C. Liverani. Non-perturbative approach to random walk in Markovian environment. Electron. Commun. Probab. 14 (2009), 245-251.

[Du] R. Durrett. Probability: Theory and Examples (4th Edition), Cambridge University Press, 2010.

[EK] S. Ethier and T. Kurtz. Markov processes, Wiley Series in Probability and Mathematical Statistics, New York, 1986.

[F] T. Funaki. Stochastic Interface Models. Ecole d' été de probabilités de Saint Flour 2003. Lecture Notes in Mathematics, 1869. Springer-Verlag, Berlin, 2005.

[FS] T. Funaki, H. Spohn. Motion by mean curvature from the Ginzburg-Landau $\nabla \varphi$ interface models. Commun. Math. Phys. 185 (1997), 1-36.

[GOS] G. Giacomin, S. Olla and H. Spohn. Equilibrium fluctuations for $\nabla \varphi$ interface model. Ann. Probab. 29 (2001), 1138-1172.

[HS] B. Helffer and J. Sjöstrand. On the correlation for Kac-like models in the convex case. J. Stat. Phys. 74 (1994), 349-409.

[JR] M. Joseph and F. Rassoul-Agha. Almost Sure Invariance Principle for ContinuousSpace Random Walk in Dynamic Random Environment. ALEA, Lat. Am. J. Probab. Math. Stat. 8 (2011), 43-57.

[KLO] T. Komorowski, C. Landim and S. Olla. Fluctuations in Markov processes. Time symmetry and martingale approximation. Grundlehren der Mathematischen Wissenschaften, 345. Springer, Heidelberg, 2012.

[Ma] P. Mathieu. Quenched invariance principles for random walks with random conductances. J. Stat. Phys. 130 (2008), no. 5, 1025-1046.

[MW] M. Maxwell and M. Woodroofe. Central limit theorems for additive functionals of Markov chains. Ann. Probab. 28 (2000), 713-724.

[Mou] J.-C. Mourrat. Variance decay for functionals of the environment viewed by the particle. Ann. Inst. Henri Poincaré Probab. Stat. 47 (2011), no. 1, 294-327.

[RS1] F. Rassoul-Agha and T. Seppäläinen. An almost sure invariance principle for random walks in a space-time random environment. Probab. Theory Related Fields 133 (2005), no. 3, 299-314. 
[RS2] F. Rassoul-Agha and T. Seppäläinen. Almost sure functional central limit theorem for ballistic random walk in random environment. Ann. Inst. H. Poincaré Probab. Statist. 45 (2009), no. 2, 373-420.

[RV] F. Redig and F. Völlering, Limit theorems for random walks in dynamic random environment. Preprint, available at arXiv:1106.4181v2.

[Ru] W. Rudin. Functional Analysis. McGraw-Hill Series in Higher Mathematics, 1973.

[SS] V. Sidoravicius and A.-S. Sznitman. Quenched invariance principles for walks on clusters of percolation or among random conductances. Probab. Theory Rel. Fields 129 (2004), no. 2, 219-244.

SA: Institut für Angewandte Mathematik

Rheinische Friedrich-Wilhelms-Universität Bonn

Endenicher Allee 60, 53115 Bonn, Germany.

andres@iam.uni-bonn.de 